\title{
ELECTROMAGNETIC ELECTRON- POSITRON PAIR PRODUCTION IN HEAVY-ION COLLISIONS WITH IMPACT PARAMETER ZERO
}

\author{
K. Hencken*, D. Trautmann* and G. Baur \\ IKP (Theorie), Forschungszentrum Jülich GmbH., \\ 5170 Jülich, Germany \\ *Inst. für theor. Physik, Univ. Basel, \\ Klingelbergstr. 82, 4056 Basel, Switzerland
}

$\mathrm{KFA}-\mathrm{IKP}(\mathrm{TH})-1993-25$ 


\title{
Electromagnetic Electron-Positron Pair Production in Heavy-Ion Collisions with Impact Parameter Zero
}

\author{
Kai Hencken, Dirk Trautmann \\ Institut für theoretieche Physik der Universität Basel, Klingelbergstr. 82, 1056 Basel, Svitzerland \\ Gerhard Baur \\ Institut fïr Kernphysik (Theorie), Forechungszentrum Jülich, 52425 Jülich, Germany \\ (Auguat 30, 1993)
}

\begin{abstract}
We present a calculation of the socond order process of electron-positron pair creation by the electromagnetic fields of two relativistic heary ions for impact parameter b sero. Total probabilities as well as differential ones are presented. A comparison with the results of the double equivalent photon appraximation (DEPA) ahows explicitly the inapplicability of this approximation in the case of total probabilities. We discuss creation of pairs with large invariant mass. The DEPA results are too high, which can be traced back to the mass singularity of the photo crose section at very amall angles, giving a discrepancy, whereas the agreement between our result and DEPA is good for larger angles.
\end{abstract}

12.20.-m;34.10. $+x ; 14.60 . C d$ 


\section{INTRODUCTION}

We investigate the electromagnetic production of electron-positron pairs in the collision of two relativistic heavy ions. The interest in this is mainly based on the fact, that the impact parameter dependent total probability of the pair creation in lowest order exceeds one for realistic accelerator parameters, so that higher order effects - especially the multiple pair production - may be of importance [1-3]. These probabilities were mainly calculated using the 20 called "equivalent photon approximation" (EPA) also known as WeizsackerWilliams method $[4,5]$. This approximation is only justified for impact parameter $b$ larger than the Compton-wavelength of the electron. As the probability increases with smaller $b$, better calculations in this area are needed. Therefore DEPA (double equivalent photon epproximation) was proposed, to do the calculations. But DEPA needs a cut-off parameter too, whose value is not given a priori. An exact treatment of the pair production should give some incight into the applicability of the DEPA.

In Sec. II we derive the matrix element for the pair production by an external field in second order. We use the field of two colliding heavy ions with impact parameter $b$ - with and without form factor - and then specialize to the case $b=0$. In Sec. III this matrix element is further reduced and the Feynman integrals occurring in it are solved analytically. This gives we the differential probability $P\left(p_{+}, p_{-}\right)$. In Sec. IV, we derive the corresponding result for the DEPA. In Sec. V, we discuss the total probability $P_{\text {total }}$. Results for some realistic heavy-ion accelerators are presented. We compare the total probability of our calculation with DEPA. We find, that the DEPA result exceeds our result, as soon as the parameter $\Lambda$ controlling the width of the form factor gets larger than the electron mass. In Sec. VI, we show results of our calculation for a number of single-differential probabilities.

It has been argued, that DEPA should again be usable for pairs with large invariant mass. In Sec. VII, we discuss this special case, finding, that there is a discrepancy between our results and DEPA. The reason for this is the mass singularity of the photo cross section at very small angles, whereas for larger angles we find good agreement with DEPA.

In the future, more calculations will be done, in order to study systematically the dependence of the probability on the different parameters $\left(m_{e}, \Lambda, \gamma, b\right)$ in different sections of the phase space.

In the appendix we summarize how to calculate the Feynman integrals occurring in our calculation and discuse the two form factors for the heavy ions, we use in our calculations.

Throughout this paper, we use the following convention: the metric is $(1,-1,-1,-1)$, and the Dirac spinors are normalized as

$$
\bar{u}_{r}(p) u_{\lrcorner}(p)=2 m \delta_{r e}, \quad \bar{v}_{r}(p) v_{\lrcorner}(p)=-2 m \delta_{r \rho} .
$$

The 4-dimensional Fourier transformation is chosen as

$$
\begin{gathered}
f\left(x^{\mu}\right)=\int \frac{d^{4} p}{(2 \pi)^{4}} \hat{f}\left(p^{\mu}\right) \exp \left(-i p^{\nu} x_{\nu}\right) \\
\hat{f}\left(p^{\mu}\right)=\int d^{4} x f\left(x^{\mu}\right) \exp \left(i p^{\nu} x_{\nu}\right),
\end{gathered}
$$

to be consistent with the decomposition into free solutions. Since we write the wave equation of the electromagnetic potential as 


$$
\square A^{\mu}(x)=j^{\mu}(x)
$$

the fine structure constant has to be defined as $\alpha:=e^{2} /(4 \pi)$.

\section{THE MATRIX ELEMENT IN SECOND ORDER}

We describe the interaction with the heavy ions as an interaction with the external field produced by both ions. We restrict ourself to lowest order, which for this process is the second. This has been studied for the first time by Landau and Lifshitz $[6]$, see also $[7,8]$. The process is shown in Fig. 1. Its matrix element is

$$
\begin{aligned}
M & =-i e^{2} \bar{u}\left(p_{-}\right) \\
& \times \int \frac{d^{4} p}{(2 \pi)^{4}}\left(p_{-}-p\right) \frac{p+m}{p^{2}-m^{2}} A\left(p_{+}+p\right) v\left(p_{+}\right) \\
& =: \bar{u}\left(p_{-}\right) \hat{M} v\left(p_{+}\right) .
\end{aligned}
$$

Here we have introduced the matrix element without Dirac spinors $\hat{M}$. From this the unpolarised differential probability to produce an electron with momentum $p_{-}$and a poritron with $p_{+}$is given by

$$
\begin{aligned}
P & =\sum_{-+-}|M|^{2} \frac{d^{3} p_{+} d^{3} p_{-}}{4 \epsilon_{+} \epsilon_{-}(2 \pi)^{6}} \\
& =\operatorname{Tr}\left(\left(t_{-}+m\right) \hat{M}\left(p_{+}-m\right) \hat{M}\right) \frac{d^{3} p_{+} d^{3} p_{-}}{4 \epsilon_{+} \epsilon_{-}(2 \pi)^{6}}
\end{aligned}
$$

using the standard method to rewrite the polarisation summation as a trace.

The external electromagnetic field is produced by the two heavy ions, i.e. we neglect the effect of the process on the ions itself, as well as the Coulomb repulsion of the ions on each other. The heavy ions are then moving on a straight line. The potential of a point charge $Q$, moving along a straight line with 4-velocity $u^{\mu}$ and at distance $r$ from the origin, is

$$
A^{\mu}(q)=-2 \pi Q u^{\mu} \frac{1}{q^{2}} \delta(q u) \exp (i q r)
$$

As we will see later, the total probability to produce a pair diverges, if we use a point charge. We therefore introduce a form factor, to describe the extended charge distribution of the heavy ion:

$$
A^{\mu}(q)=-2 \pi Q u^{\mu} \frac{F\left(q^{2}\right)}{q^{2}} \delta(q u) \exp (i q r)
$$

The form factors used throughout the calculations are the dipole form factor

$$
F_{\text {dipole }}\left(q^{2}\right)=\frac{\Lambda^{2}}{\Lambda^{2}-q^{2}}
$$

and a form factor, which is the sum of two dipole form iactors 


$$
F_{\text {dowbe }}\left(q^{2}\right)=c_{1} \frac{\Lambda_{1}^{2}}{\Lambda_{1}^{2}-q^{2}}+c_{2} \frac{\Lambda_{2}^{2}}{\Lambda_{2}^{2}-q^{2}},
$$

called by us the "double dipole formfactor".

In App. B we show how to choose the parameters in the form factors. The dipole form factor is that of a Yukawa charge distribution, which is surely not very realistic. On the other hand, the probabilities are normally not very sensitive to the detailed form of the form factor, and we can treat $\dot{M}$ analytically using (8) and (9).

The field produced by both ions is then

$$
\begin{aligned}
A_{\mu}(q) & =-2 \pi e \frac{F\left(q^{2}\right)}{q^{2}}\left(Z_{1} u_{\mu}^{(1)} \delta\left(q u^{(1)}\right) \exp (i q b / 2)\right. \\
& \left.+Z_{2} u_{\mu}^{(2)} \delta\left(q u^{(2)}\right) \exp (-i q b / 2)\right) .
\end{aligned}
$$

In the following, we consider only symmetric configurations, where both ions are identical: $Z=Z_{1}=Z_{2}$ and choose the c.m. system, so that $\gamma=\gamma_{1}=\gamma_{2}$. This correspond to $\gamma_{L}=2 \gamma^{2}-1$ for one ion in the laboratory system of a fixed target machine. We will neglect at the moment the form factor $F\left(q^{2}\right)$, as its inclusion in the formulas is straightforward, but come back to it at the end.

With this potential, there are four possible combinations in $\hat{M}$, but only two of them are allowed kinematically (see Fig. 2). Therefore we have:

$$
\begin{aligned}
\hat{M} & =-i e^{2}\left(\int \frac{d^{4} p}{(2 \pi)^{4}} f^{(1)}\left(p_{-}-p\right) \frac{p+m}{p^{2}-m^{2}} f^{(2)}\left(p_{+}+p\right)+\int \frac{d^{4} p}{(2 \pi)^{4}} A^{(2)}\left(p_{-}-p\right) \frac{p+m}{p^{2}-m^{2}} f^{(1)}\left(p_{+}+p\right)\right) \\
& =-i\left(\frac{Z e^{2}}{2 \pi}\right)^{2}\left(x^{(1)} \int d^{4} p \frac{p+m}{\left(p_{-}-p\right)^{2}\left(p^{2}-m^{2}\right)\left(p_{+}+p\right)^{2}} x^{(2)} \delta\left(\left(p_{-}-p\right) u^{(1)}\right) \delta\left(\left(p_{+}+p\right) u^{(2)}\right)\right. \\
& \times \exp (-i p b) \exp \left(i\left(p_{-}-p_{+}\right) b / 2\right) \\
& +\not^{(2)} \int d^{4} p \frac{\not+m}{\left(p_{-}-p\right)^{2}\left(p^{2}-m^{2}\right)\left(p_{+}+p\right)^{2}} x^{(1)} \delta\left(\left(p_{-}-p\right) u^{(2)}\right) \delta\left(\left(p_{+}+p\right) u^{(1)}\right) \\
& \left.\times \exp (+i p b) \exp \left(-i\left(p_{-}-p_{+}\right) b / 2\right)\right) .
\end{aligned}
$$

We now restrict ourself to the case, where the impact parameter $b$ is zero. In $\hat{M}, b$ only occurs in the exponent, multiplied by some transverse momente $p_{\perp}$. As we will see later in the discussion of $\boldsymbol{P}_{\text {todel }}$, mainly small $\boldsymbol{p}_{\perp}$ contribute to the total probability, smaller than $\Lambda$ (of the form factor) and also mainly amaller than $m_{e}$. The case $b=0$ can therefore be seen as the first term of an expansion in $p_{\perp} b$, which should surely be good, as long as $b$ is smaller than the nuclear radius and should not be too different for $b$ smaller than the Compton wavelength: $b<\lambda_{c}=1 / m_{e} . \hat{M}$ for $b=0$ is then

$$
\begin{aligned}
\hat{M} & =-i\left(\frac{Z e^{2}}{2 \pi}\right)^{2} \\
& \times\left(x^{(1)} \int d^{4} p \frac{p+m}{\left(p_{-}-p\right)^{2}\left(p^{2}-m^{2}\right)\left(p_{+}+p\right)^{2}} x^{(2)} \delta\left(\left(p_{-}-p\right) u^{(1)}\right) \delta\left(\left(p_{+}+p\right) u^{(2)}\right)\right. \\
& \left.+x^{(2)} \int d^{4} p \frac{\not+m}{\left(p_{-}-p\right)^{2}\left(p^{2}-m^{2}\right)\left(p_{+}+p\right)^{2}} x^{(1)} \delta\left(\left(p_{-}-p\right) u^{(2)}\right) \delta\left(\left(p_{+}+p\right) u^{(1)}\right)\right) .
\end{aligned}
$$




\section{EXACT SOLUTION OF THE MATRIX ELEMENT}

In order to find an analytical form for the matrix element, we have to integrate over the internal momentum. First we define

$$
\begin{aligned}
I_{D}^{(m, i)} & :=\int d^{4} p \frac{\left\{m, p_{i}\right\}}{\left(p_{-}-p\right)^{2}\left(p^{2}-m^{2}\right)\left(p_{+}+p\right)^{2}} \\
& \times \delta\left(\left(p_{-}-p\right) u^{(1)}\right) \delta\left(\left(p_{+}+p\right) u^{(2)}\right) \\
I_{X}^{(m, i)} & :=\int d^{4} p \frac{\left\{m, p_{i}\right\}}{\left(p_{-}-p\right)^{2}\left(p^{2}-m^{2}\right)\left(p_{+}+p\right)^{2}} \\
& \times \delta\left(\left(p_{-}-p\right) u^{(2)}\right) \delta\left(\left(p_{+}+p\right) u^{(1)}\right)
\end{aligned}
$$

which are the integrals occurring in the "direct" and "exchanged" diagram, giving for $\hat{M}$

$$
\begin{aligned}
\hat{M} & =-i\left(\frac{Z e^{2}}{2 \pi}\right)^{2}\left(x^{(1)}\left(f_{D}^{(i)}+I_{D}^{(m)}\right) \not^{(2)}\right. \\
& \left.+\mu^{(2)}\left(I_{X}^{(i)}+I_{X}^{(m)}\right) \not^{(1)}\right) .
\end{aligned}
$$

Now we use the two delta functions to reduce the 4-dimensional integration. The 4-velocities $u^{(1)}$ and $u^{(2)}$ in the c.m. frame are

$$
\begin{aligned}
& u^{(1)}=\gamma(1,0,0, \beta)=: \gamma w^{(1)} \\
& u^{(2)}=\gamma(1,0,0,-\beta)=: \gamma w^{(2)} .
\end{aligned}
$$

Evaluating them determines the zero- and $z$-component of the internal momentum. In the case " $D$ ", they are

$$
\begin{aligned}
\epsilon=\epsilon_{D} & :=\frac{1}{2}\left(\epsilon_{-}-\epsilon_{+}\right)-\frac{1}{2} \beta\left(p_{-x}+p_{+z}\right) \\
p_{z}=p_{D} & :=\frac{1}{2}\left(p_{-z}-p_{+x}\right)-\frac{1}{2 \beta}\left(\epsilon_{-}+\epsilon_{+}\right)
\end{aligned}
$$

and in the case " $\mathrm{X}$ ":

$$
\begin{aligned}
\epsilon=\epsilon_{X} & :=\frac{1}{2}\left(\epsilon_{-}-\epsilon_{+}\right)+\frac{1}{2} \beta\left(p_{-z}+p_{+z}\right) \\
p_{z}=p_{X} & :=\frac{1}{2}\left(p_{-z}-p_{+z}\right)+\frac{1}{2 \beta}\left(\epsilon_{-}+\epsilon_{+}\right) .
\end{aligned}
$$

The only difference between both formulas is the sign between the two terms, which comes from the exchange of $\beta \leftrightarrow-\beta$ in the four-velocities.

We now split $p$ into its longitudinal and transverse part defining

$$
p_{D I}:=\left(\epsilon_{D}, 0,0, p_{D}\right), \quad p_{X I}:=\left(\epsilon_{X}, 0,0, p_{X}\right)
$$

and the same with $p_{-}$and $p_{+}$. The integrals $I_{D}^{(m, i)}$ and $I_{X}^{(m, i)}$ are now 


$$
\begin{aligned}
I_{D}^{(m, i)} & =\frac{1}{2 \gamma^{2} \beta} \int d^{2} p_{\perp} \\
& \times \frac{\left\{m, p_{i}\right\}}{\left(\left(p_{-1}-p_{D I}\right)^{2}+\left(p_{-\perp}-p_{\perp}\right)^{2}\right)\left(p_{D I}^{2}+p_{\perp}^{2}-m^{2}\right)\left(\left(p_{+l}+p_{D I}\right)^{2}+\left(p_{+\perp}+p_{\perp}\right)^{2}\right)} \\
I_{X}^{(m, i)} & =\frac{1}{2 \gamma^{2} \beta} \int d^{2} p_{\perp} \\
& \times \frac{\left\{m, p_{i}\right\}}{\left(\left(p_{-1}-p_{X I}\right)^{2}+\left(p_{-\perp}-p_{\perp}\right)^{2}\right)\left(p_{X I}^{2}+p_{\perp}^{2}-m^{2}\right)\left(\left(p_{+l}+p_{X I}\right)^{2}+\left(p_{+\perp}+p_{\perp}\right)^{2}\right)} .
\end{aligned}
$$

(Note that $p_{\perp}$ only has spatial components, evaluating the scalar products gives then an extra minus sign). The factor $\frac{1}{2 \gamma^{2} \beta}$ comes from the evaluation of the delta functions. The difference between the two integrals is only the substitution of $p_{D X}$ with $p_{X I}$.

Examining $I_{D}$ and $I_{X}$ for $(m),(0)$ and $(z)$, we find, that the numerator of the integrand does not depend on $p_{\perp}$ and therefore we can rewrite them as

$$
\begin{aligned}
I_{D}^{(m, 0, x)} & =\frac{1}{2 \gamma^{2} \beta}\left\{m, \epsilon_{D}, p_{D}\right\} \int d^{2} p_{\perp} \\
& \times \frac{1}{\left(\left(p_{-l}-p_{D I}\right)^{2}+\left(p_{-\perp}-p_{\perp}\right)^{2}\right)\left(p_{D I}^{2}+p_{\perp}^{2}-m^{2}\right)\left(\left(p_{+l}+p_{D I}\right)^{2}+\left(p_{+\perp}+p_{\perp}\right)^{2}\right)}
\end{aligned}
$$

and the corresponding result for $I_{X}^{(m, 0, x)}$. As we thow in App. A, the integral on the right side is just one of the standard integrals, the acalar three-term-integral. Therefore we can write

$$
\begin{aligned}
I_{D}^{(m, 0, x)} & =-\frac{1}{2 \gamma^{2} \beta}\left\{m, \epsilon_{D}, p_{D}\right\} \\
& \times I^{S}\left(k_{2 D}, k_{3 D}, m_{1 D}^{2}, m_{2 D}^{2}, m_{3 D}^{2}\right) \\
& =:-\frac{1}{2 \gamma^{2} \beta}\left\{m, \epsilon_{D}, p_{D}\right\} I_{D}^{S}
\end{aligned}
$$

with

$$
\begin{aligned}
& k_{2 D}=-p_{-1}, \\
& k_{3 D}=p_{+1}, \\
& m_{1 D}^{2}=m^{2}-p_{D l}^{2}, \\
& m_{2 D}^{2}=-\left(p_{D l}-p_{-1}\right)^{2}, \\
& m_{3 D}^{2}=-\left(p_{D l}+p_{+1}\right)^{2}
\end{aligned}
$$

and the same for $I_{X}^{(m, 0, x)}$ by changing the index to " $\mathrm{X}$ ".

On the other hand, for $(x)$ and $(y)$, the numerator depends on $p_{\perp}$, over which we have to integrate. This integral is the simplest type of a so-called tensor integral, and it is clear, that it can be split into two parts:

$$
I_{D}^{(x, y)}=-\frac{1}{2 \gamma^{2} \beta}\left(-p_{-\perp} I_{D}^{2}+p_{+\perp} I_{D}^{3}\right)
$$

and the corresponding result for $I_{X}^{(s, y)} . I_{D}^{2}$ and $I_{D}^{3}$ are again standard Feynman integral found in App. A: 


$$
I_{D}^{(2,3)}=I^{(2,3)}\left(k_{2 D}, k_{3 D}, m_{1 D}^{2}, m_{2 D}^{2}, m_{3 D}^{2}\right)
$$

and the corresponding for $I_{X}^{(2,3)}$.

We finally get

$$
\begin{aligned}
\hat{M} & =i \frac{2(Z \alpha)^{2}}{\beta}\left(\psi^{(1)}\left(\phi_{D}+m\right) \psi^{(2)} I_{D}^{S}\right. \\
& -\psi^{(1)} f_{-\perp} \psi^{(2)} I_{D}^{2}+\psi^{(1)} \psi_{+\perp} \psi^{(2)} I_{D}^{3} \\
& +\psi^{(2)}\left(\phi_{X 1}+m\right) \psi^{(1)} I_{X}^{S} \\
& \left.-\psi^{(2)} \psi_{-\perp} \psi^{(1)} I_{X}^{2}+\psi^{(2)} \psi_{+\perp} \psi^{(1)} I_{X}^{3}\right) .
\end{aligned}
$$

An alternative form for $\hat{M}$ can be found, by using the properties of the Dirac spinors:

$$
\begin{aligned}
& \left(p_{+}+m\right) v\left(p_{+}\right)=0, \\
& \bar{u}\left(p_{-}\right)\left(p_{-}-m\right)=0 .
\end{aligned}
$$

Rewriting them as

$$
\begin{aligned}
& x_{+\perp} v\left(p_{+}\right)=-\left(p_{+1}+m\right) v\left(p_{+}\right) \\
& \bar{u}\left(p_{-}\right) p_{-\perp}=-\bar{u}\left(p_{-}\right)\left(p_{-1}-m\right),
\end{aligned}
$$

we get

$$
\begin{aligned}
\dot{M} & =i \frac{2(Z \alpha)^{2}}{\beta}\left(\psi^{(1)}\left(\phi_{D l}+m\right) \psi^{(2)} I_{D}^{S}\right. \\
& -\left(\phi_{-l}-m\right) \psi^{(1)} \psi^{(2)} I_{D}^{2}+\psi^{(1)} \psi^{(2)}\left(\phi_{+l}+m\right) I_{D}^{3} \\
& +\psi^{(2)}\left(\phi_{X I}+m\right) \psi^{(1)} I_{X}^{S}-\left(\phi_{-l}-m\right) \psi^{(2)} \psi^{(1)} I_{X}^{2} \\
& \left.+\psi^{(2)} \psi^{(1)}\left(\psi_{+l}+m\right) I_{X}^{3}\right) .
\end{aligned}
$$

This form for $\hat{M}$ has been used to test the correctness of our calculations, ws well as its numerical stability.

Let us now come back to the inclusion of the form factor $F\left(q^{2}\right)$. It is easy to see, that this only changes the form of $I_{D}$ and $I_{X}$ to

$$
\begin{aligned}
I_{D}^{(m, i)} & =\frac{1}{2 \gamma^{2} \beta} \int d^{2} p_{\perp}\left\{m, p_{i}\right\} \\
& \times\left.\frac{F\left(\left(p_{-}-p\right)^{2}\right) F\left(\left(p_{+}+p\right)^{2}\right)}{\left(p_{-}-p\right)^{2}\left(p^{2}-m^{2}\right)\left(p_{+}+p\right)^{2}}\right|_{p_{l}=p_{D}} \\
I_{X}^{(m, i)} & =\frac{1}{2 \gamma^{2} \beta} \int d^{2} p_{\perp}\left\{m, p_{i}\right\} \\
& \times\left.\frac{F\left(\left(p_{-}-p\right)^{2}\right) F\left(\left(p_{+}+p\right)^{2}\right)}{\left(p_{-}-p\right)^{2}\left(p^{2}-m^{2}\right)\left(p_{+}+p\right)^{2}}\right|_{p_{l}=p_{X}} .
\end{aligned}
$$


Now we can use the property of the dipole form factor:

$$
\frac{F\left(q^{2}\right)}{q^{2}}=\frac{\Lambda^{2}}{\left(\Lambda^{2}-q^{2}\right) q^{2}}=\frac{1}{q^{2}}+\frac{1}{\Lambda^{2}-q^{2}}
$$

which allows us to rewrite the integrals as the sum and difference of four integrals of the type, we have already solved in terms of the elementary Feynman integrals. The scalar and tensor-integrals corresponding to (21) and (24) are:

$$
\begin{aligned}
& I_{D}^{(S, 2,3)}=I^{(S, 2,3)}\left(k_{2 D}, k_{3 D}, m_{1 D}^{2}, m_{2 D}^{2}, m_{3 D}^{2}\right) \\
& -I^{(S, 2,3)}\left(k_{2 D}, k_{3 D}, m_{1 D}^{2}, m_{2 D}^{2}+\Lambda^{2}, m_{3 D}^{2}\right) \\
& -I^{(S, 2,3)}\left(k_{2 D}, k_{3 D}, m_{1 D}^{2}, m_{2 D}^{2}, m_{3 D}^{2}+\Lambda^{2}\right) \\
& +I^{(S, 2,3)}\left(k_{2 D}, k_{3 D}, m_{1 D}^{2}, m_{2 D}^{2}+\Lambda^{2}, m_{3 D}^{2}+\Lambda^{2}\right)
\end{aligned}
$$

which we use in formula (25) and (28) for $\hat{M}$.

The anme can be done with the double dipole form factor (sce also App. B)

$$
\frac{F\left(q^{2}\right)}{q^{2}}=\frac{1}{q^{2}}+c_{1} \frac{1}{\Lambda_{1}^{2}-q^{2}}+c_{2} \frac{1}{\Lambda_{2}^{2}-q^{2}}
$$

giving a sum of total nine standard Feynman integrals.

$\hat{M}$ is now used in order to calculate the Trace $\operatorname{Tr}\left(\left(\phi_{-}+m\right) \hat{M}\left(f_{+}-m\right) \hat{\vec{M}}\right)$. For this tedious, but straightforward calculation, we have used a symbolic calculation program (FORM [9]).

Some care has been taken in evaluating these long expressions numerically, in order to avoid some of the large cancellations, which normally occur in the scalar products. That these cancellation do occur can be shown e.g. in $m_{2 D}^{2}$, where they can be seen very easily:

$$
\begin{aligned}
m_{2 D}^{2} & =-\left(p_{D l}-p_{-l}\right)^{2}=\left(p_{D}-p_{-z}\right)^{2}-\left(\epsilon_{D}-\epsilon_{-}\right)^{2} \\
& =\frac{1}{4} \frac{1}{\gamma^{2} \beta^{2}}\left(\left(\epsilon_{-}+\epsilon_{+}\right)+\beta\left(p_{-x}+p_{+z}\right)^{2},\right.
\end{aligned}
$$

where we have used the fact, that $1-\beta^{2}=\gamma^{-2}$. We see, that for large values of $\gamma, m_{2 D}^{2}$ becomes very small, which means, that the cancellations in the first expressions are very large.

To avoid these cancellations in the scalar products, we did not use the longitudinal parts of the 4-vectors directly, but transformed them into light-cone variables. Longitudinal vectors we have, are $w^{(1)}, w^{(2)}, p_{+l}, p_{-l}, p_{I D}$ and $p_{l X}$, transverse vectors only $p_{+1,} p_{-1}$.

For the light cone variables, we define a 't'- and a '-'-component of an arbitrary vector ข:

$$
v_{+^{\prime}}=v_{0}+v_{x}, \quad v_{-^{\prime}}=v_{0}-v_{z} .
$$

The scalar products between longitudinal vectors is then

$$
\begin{aligned}
(v, w) & =\frac{1}{2}\left(v_{l^{\prime}} w_{I_{-}}+v_{\iota_{-}} w_{l^{\prime}}\right) \\
(v, v) & =v_{+^{\prime}} v_{\iota^{\prime}} .
\end{aligned}
$$


Generally, one of the variables ' + ', ' - ' is small, the other large. For example, $w_{++^{\prime}}^{(1)}=1+\beta$ is large, $w_{-\prime}^{(1)}=1-\beta$ is very small, because $\beta$ is close to one. We calculate it, using

$$
w_{-^{\prime}}^{(1)}=1-\beta=\frac{(1-\beta)(1+\beta)}{(1+\beta)}=\frac{1}{\gamma^{2} w_{+^{\prime}}^{(1)}} .
$$

The same can be done with the other vectors, e.g. if $p_{+x}, p_{-x}>0$, we calculate $p_{+, f^{\prime}}$ and $p_{-,{ }^{\prime}+}$ directly and the other as

$$
\begin{aligned}
& p_{+,{ }^{\prime}-}=\frac{\epsilon_{+}^{2}-p_{+z}^{2}}{\epsilon_{+}+p_{+z}}=\frac{m^{2}+p_{+\perp}^{2}}{p_{+,^{\prime}+{ }^{\prime}}} \\
& p_{-,{ }^{\prime}}=\frac{\epsilon_{-}^{2}-p_{-\varepsilon}^{2}}{\epsilon_{-}+p_{-\Sigma}}=\frac{m^{2}+p_{-\perp}^{2}}{p_{-,{ }^{\prime}}} \text {. }
\end{aligned}
$$

\section{PAIR PRODUCTION IN DOUBLE EQUTVALENT PHOTON APPROXIMATION}

The equivalent photon approximation (EPA) or Weizeäcker-Williams method has been used in the past, to calculate electromagnetic processes in heavy-ion collisions $[4,5]$. It consists of replacing the eloctromagnetic field of the fast moving ion by a spectrum of real photons. Then one folds the photo cross section with the number of equivalent photons $N(\omega)$, to get the cross section for the heavy-ion process. In the double equivalent photon approximation (DEPA), one replaces the field of both ions by equivalent photons, then folds with both photon distributions. Normally, one uses the total equivalent photon number, the one integrated over all impact parameters $[10,11]$. Recently also the b-dependent DEPA has been investigated by Baur and Ferreira Filho [12,13] and also by Vidović et. al. [14]. One of the problems of the DEPA is - because the virtual photons with $q^{2} \neq 0$ are all replaced by real photons - we have to introduce a cutoff in $q^{2}$, to avoid the logarithmic divergence, even though the main contribution comes from that part, where $q^{2}$ is small. The cutoff can also be interpreted a a cut-off in the impact parameter (in the case of the EPA, where only one ion is replaced by the photan spectrum), or a cut off in the distance between the ion and the place, where the interaction with the electron or positron takes place. Also contributions coming from scalar photons are neglecteo. There has been some discuscion about the chaice of the cutoff. On the one hand, the form factor of the ion decreases the number of photons with large $q^{2}$, on the other hand, the matrix element decreases, if the momentum of the internal eloctron line $|p|$ is greater than $m_{e}$, therefore, we are not allowed to replace this matrix element with the one for the photo process (see e.g. the discussion in Sec. 6 in [11]).

The DEPA gives us on the one hand an independent check for the correctnes of our results and on the other hand a test of the applicability of the DEPA in this case, especially to see, if the cutoff is given by the form factor.

For our calculation, we use the formula given by Baur and Ferreira Filho [12]. For $b=0$ their result is:

$$
d P(b=0)=\frac{d \omega_{1}}{\omega_{1}} \frac{d \omega_{2}}{\omega_{2}} \int d^{2} \rho N\left(\omega_{1}, \rho\right) N\left(\omega_{2}, \rho\right) \sigma_{\|}\left(\omega_{1}, \omega_{2}\right),
$$


where $\sigma_{\|}$is the total cross section for pair production with the two photons with parallel polarisation. $N(\omega, p)$ is the $p$-dependent equivalent photon number. In the ultrarelativistic limit it is given by:

$$
N(\omega, \rho)=\frac{Z^{2} \alpha}{\pi^{2}} \frac{\phi(x, \rho)}{\rho^{2}}
$$

with $x:=\frac{\omega p}{r c}$ and

$$
\phi(x, \rho)=\left|\int_{0}^{\infty} d u u^{2} J_{1}(u) \frac{F\left(-\frac{x^{2}+u^{2}}{\rho^{2}}\right)}{x^{2}+u^{2}}\right|^{2} .
$$

With a dipole form factor (Eq. 8), we are splitting again $F\left(-\left(x^{2}+u^{2}\right) / \rho^{2}\right) /\left(x^{2}+u^{2}\right)$ into two parts:

$$
\frac{\rho^{2} \Lambda^{2}}{\rho^{2} \Lambda^{2}+x^{2}+u^{2}} \frac{1}{x^{2}+u^{2}}=\frac{1}{x^{2}+u^{2}}-\frac{1}{\rho^{2} \Lambda^{2}+x^{2}+u^{2}}
$$

rewriting $\phi$ as:

$$
\begin{aligned}
\phi(x, \rho) & =\mid \int_{0}^{\infty} d u u^{2} J_{1}(u) \\
& \times\left.\left(\frac{1}{x^{2}+u^{2}}-\frac{1}{\rho^{2} \Lambda^{2}+x^{2}+u^{2}}\right)\right|^{2}
\end{aligned}
$$

The integral

$$
\int_{0}^{\infty} d u u^{2} J_{1}(u) \frac{1}{z^{2}+u^{2}}=z K_{1}(z)
$$

can be solved analytically, giving for $\phi$ the result for a point-source, which is known. Therefore $\phi(x, \rho)$ is given by

$$
\phi(x, \rho)=\left|x K_{1}(x)-\sqrt{\rho^{2} \Lambda^{2}+x^{2}} K_{1}\left(\sqrt{\rho^{2} \Lambda^{2}+x^{2}}\right)\right|^{2}
$$

and using the definition of $x$, we find

$$
\begin{aligned}
N(\omega, \rho) & =\frac{Z^{2} \alpha}{\pi^{2}} \mid \frac{\omega}{\gamma} K_{1}\left(\frac{\omega}{\gamma} \rho\right) \\
& -\left.\sqrt{\frac{\omega^{2}}{\gamma^{2}}+\Lambda^{2}} K_{1}\left(\sqrt{\frac{\omega^{2}}{\gamma^{2}}+\Lambda^{2} \rho}\right)\right|^{2} .
\end{aligned}
$$

The cross section for real photons is [11]

$$
\begin{aligned}
\sigma_{\|} & =\frac{4 \pi \alpha^{2}}{s}\left(\left(1+\frac{4 m^{2}}{s}-\frac{12 m^{4}}{s^{2}}\right) L\right. \\
& \left.-\left(\frac{1}{s}+\frac{6 m^{2}}{s^{2}}\right) \Delta t\right)
\end{aligned}
$$


with

$$
\begin{aligned}
s & =4 \omega_{1} \omega_{2} \\
\Delta t & =s \sqrt{1-\frac{4 m^{2}}{s}} \\
L & =2 \log \left(\frac{\sqrt{s}}{2 m}+\sqrt{\frac{s}{4 m^{2}}-1}\right) .
\end{aligned}
$$

For the total probability we integrate over $d \omega_{1}, d \omega_{2}$ and $d^{2} \rho$ using a Monte-Carlo integration routine (VEGAS $[15,16])$. The integration variables, we use, are $\log \rho, \log \omega_{2}$ and $\log s$ and the boundaries of the integral have been increased, until the result does not change.

\section{The TOTAL PROBability $\boldsymbol{P}_{\text {todal }}$}

The differential probability is now integrated over all six momentum variables. For this we used again the Monte-Carlo integration routine [15,16]. The integration over one of the angle is trivial, an integration over 5 variables remains to be done, for which we used $t_{3}, t_{1}, \phi, \eta$ and $x$. The momentum of electron and pocitron expressed in these variables are:

$$
\begin{aligned}
& p_{+z}=\left(\exp \left(t_{x}\right)-1\right) \cos \eta \\
& p_{-x}=\left(\exp \left(t_{x}\right)-1\right) \sin \eta, \\
& p_{+1}=\left(\exp \left(t_{\perp}\right)-1\right) \cos \chi(1,0), \\
& p_{-1}=\left(\exp \left(t_{1}\right)-1\right) \sin \chi(\cos \phi, \sin \phi) .
\end{aligned}
$$

The integration boundaries in $t_{z}$ and $t_{\perp}$ were incremented, until the MC-integral converges. A good estimate for the boundaries is given by $\log \left(\gamma \max \left(\Lambda, m_{e}\right)+1\right)$ for $t_{x}$ and by $\log \left(\max \left(\Lambda, m_{e}\right)+1\right)$ for $t_{\perp}$. $\Lambda$ was chosen according to App. B as $\Lambda=83 \mathrm{MeV}$ for the dipole form factor and similarly for the double dipole form factor. In this section we will only discuss the probability divided by $(Z \alpha)^{4}, a$ this is a common factor in our formula.

Fig. 3 shows the dependence of the total probability on $\Lambda$ for two different velues of $\gamma$ (100 and 3400). We see, that there is a logarithmic dependence on $\Lambda, s 0$ that the result seems to be divergent for a point charge. This divergence is of course no contradiction to the fact, that the total croes section for a point charge

$$
\sigma=\int 2 \pi b d b P(b)
$$

is finite.

Fig. 4 shows $P_{\text {todal }} /(Z \alpha)^{4}$ as a function of $\gamma$. We use the dipole form factor as well as the double dipole form factor. The difference between both is very umall. This confirms our assumption, that the detailed form of the form factor is not important, as only small $q^{2}$ contribute considerably to the total probability. Together with our calculation, we aleo plot the results of the EPA calculation for different values of the impact parameter $b$ (formula 7.3.10 in [10], where we have neglected the term with $\bar{f}$ ). One sees, that our calculation for $b=0$ only increases linearly with $\log \gamma$, whereas the EPA result increases with $(\log \gamma)^{2}$. Even for $b \approx \lambda_{c}$ the EPA result is larger than our result for $b=0$. As the probability should 
always increase with smaller impact parameter, we conclude, that the EPA result can not be used for small impact parameter and that the range, where it is not applicable, even increases slowly for larger value of $\gamma$. This result is of course well known, therefore (7.3.10) has always be used only for impact parameter $b>\lambda_{c}$.

We aleo note, that the total probabilities are not very large. Values smaller than 35 have to be multiplied by $(Z \alpha)^{4}<.2$. Therefore the total probability is smaller than 7 for realistic parameters.

Fig. 5 shows again the total probability, but now together with those for $\mu$ - and $r$-pairs. Their probabilities are much amaller due to the larger mass of the $\mu$ and the $\tau$.

Finally in Trble I we give our predictions for the total probability for the electron-positron pair production for some heavy-ion accelerators.

We now compare the calculation with the DEPA result. Now each of the ions has its own form factor independent of the other, in order to study the influence of the individual form factor on the total probability.

In Fig. 6 we compare our calculation with DEPA at RHIC-energies $(\gamma=100)$. We show $P_{\text {todal }} /(Z \alpha)^{4}$ as a function of $\Lambda_{1}$ (the parameter controlling the width of one dipole form factor), $\Lambda_{2}$ for the other ion has been kept fixed. This comparison clearly shows, that DEPA masively orereatimates the Born calculation, as soon as one and even more if both $\Lambda$ 's are larger than the electron mase. Therefore the reduction in $q^{2}$ due to the form factors does not suffice, the decrease of the matrix element of the process itself as soon as the internal momentum of the fermion gets larger than $m_{e}$ is more important. But even then, this decrease alone does not suffice, as the total probability still depends on the form factor.

We see aleo, that the probability gets independent of $\Lambda_{1}$, if it is much larger than $m_{e}$ and $\Lambda_{2}$. This corresponds to the result, that the production of a pair by a photon in the field of a nucleus is independent of the size of the nucleus, if the size is much smaller than the Compton wavelength.

\section{THE SINGLE-DIfFerential PROBabilities}

Bexide the total probability, we have also calculated some of the single-differential probabilities. For this, we have used the fact, that with a MC-integration single-differential distribution can be calculated very easily, wrting the individual integration points into bins. Therefore, one run of the program can be used, to calculate several differential probabilities at once. A dieadrantage of this method is, that a large number of sample points is needed, if we want to get some accuracy, because the result spans several orders of magnitudes. Again, as in the previous case, a common factor $(Z \alpha)^{4}$ has been extracted from $\boldsymbol{P}$.

All calculations were done for CERN SPS -,RHIC- and LHC-energies - that is for $\gamma=10,100,3400$ - waing both the simple dipole and the double dipole form factor. Generally our results show come difference for the two form factors, but only a very small one, so that our results should represent the real situation well.

Fig. 7 shows the dependence on the energy of the position (as the result is symmetric with respect to electron and positron, this is the same for the electron). The probability has the characteristic peak at low energies and decreases for higher energies. 
Fig. 8 shows the angular distribution, where $\theta$ is the angle between the momentum $p_{+}$ and the beam-axis (z-axis). For large values of $\gamma$, the particles are produced mainly at very small angles.

We also study the distributions related to the total momentum $P=p_{+}+p_{-}$. These are the invariant mase $M=\sqrt{P^{2}}$, the rapidity $Y=\frac{1}{2} \log \left(\frac{P_{0}+P_{\mathrm{f}}}{P_{0}-P_{s}}\right)$ and the transverse momentum $P_{\perp}$. They are shown in Figs. $9(P(M)), 10(P(Y))$ and $11\left(P\left(P_{\perp}\right)\right)$. We show only $P(M)$ for relatively small values of $M$. A discussion of the behavior of $P(M)$ for large invariant mass can be found in the next section.

\section{A COMPARISON WITH DEPA FOR LARGE INVARIANT MASS}

Finally we want to discuss the case, where the invariant mase of the two leptons is large. It has been argued, that in this case, the DEPA approximation, which failed at low invariant mass, should again be applicable. This is based on the fact, that in this case, the only relevant momentum acale is the invariant mase, so that one may neglect effects coming from the electron mass.

Fis. 12 shows a comparison of $P(M)$ betwoen DEPA and our calculation. Even though the difference between both is not that bad for large invariant mase, DEPA is off by about a factor of 2.

The same can be seen in Fig. 13, where we compare $P(M, Y)$ for rapidity $Y=0$.

For the calculation of $P(M, Y=0)$ we have used the fact, that for $Y=0$, (which means that $p_{+z}=-p_{-x}$ ) and with fixed $M, p_{+z}$ and $p_{-z}$ can be calculated as a function of the transverse momenta $p_{+1}$ and $p_{-\perp}$ together with their angle $\phi$. The remaining 3-dimensional integration was again done with the MC-integration routine. For the DEPA calculation, $Y=0$ and fixed $M$ means, that $\omega_{1}$ and $\omega_{2}$ are fixed at $M / 2$, only the integration over $\rho$ has to be done. As $\sigma$ is a function of $M$ alone, we can use formula (46) again, see also [17]. We see again, that the result of the DEPA is too large by a factor of 2.

This seems to be in contradiction with the arguments given above. In order to see, where the discrepancy comes from, we calculated also $P(M, Y, \Omega)$. For the Born calculation we used again the corting of $P$ into bins. For the DEPA calculation, the differential crose section for the photon-photon proceas [7] has been used, wee also [18].

We show $P(M, Y=0, \Omega)$ in Fig. 14 as a function of $\theta$, the angle of $p_{+}$with the beamaxis. For large angles DEPA and our calculations afree quite well, but for small angles the DEPA calculation is too high. As one may object, that this is an effect of the integration over the transverse moments in the DEPA, which should smear out the angular distribution, we show in Fig. $15 P\left(M, Y=0, \Omega_{\Delta}\right)$ as a function of $\theta_{\Delta}$, the angle of $\left(p_{+}-p_{-}\right)$with the beam-axis. For the DEPA, in the form used by us, the two particles are produced with their momenta exactly opposite to each other, 20 the curve is identical with the previous one (nee [17] for a better calculation, where the transverse mornentum distribution has been included in DEPA). But in our calculation, we should be able, to unfold with this approximately the traneverse momentum distribution coming from the virtual photons. Here again, we find good agreement at large angles, but DEPA is too large at amall ones.

The reason for this can be explained as follows: The total cross section for the pair production in lowest order by a photon in the electromagnetic field of a nucleus is given by the Bethe-Heitler formula [19] 


$$
\sigma=\frac{28}{9} \frac{Z^{2} \alpha^{3}}{m_{e}^{2}} \log \frac{E_{\gamma}}{m_{e}} .
$$

The logarithmic term in this formula is due to the pairs, which are produced at very small angles. This behavior is due to a 80 called mass singularity in the matrix element, the fact, that the propagator of the internal particle is very large, and even would become aingular for $m_{e} \rightarrow 0$. But this behavior is connected to the fact, that we are looking at a real photon with $q^{2}=0$. In the DEPA/EPA we neglect the dependence of the matrix element on the transverse momentum. But $a q^{2}$ is mainly given by $q_{\perp}^{2}$, this is only justified, as long as $q_{\perp}$ is smaller than $m_{e}$, whereas the form factor of the ions allows $q_{\perp}$ to be as high as $83 \mathrm{MeV}$. That is, we are not allowed to neglect $m_{e}$ compared with $M$ even for very large $M$, because setting $m_{e}=0$ would make our croses section divergent. There are always three momentum scales given by $M, \Lambda$ and $m_{e}$. The equivalent photon method has to be modified in the region of amall angles. This expleins, why the calculation of $P(M)$ and $P(M, Y=0)$ are to large, as they are integrated over the whole $\theta$-range. But as this error only showe up in - logarithmic term, the deviation of the DEPA is not that large, compared e.g. with $\boldsymbol{P}_{\text {tocal }}$, where DEPA overeatimates the result by orders of magnitude.

\section{ACKNOWLEDGMENTS}

We would like to thank Jos Vermaseren, Mario Vidović and Niels Baron for their help and advice during some stage of this calculation.

\section{APPENDIX A: THE TWO-DIMENSIONAL FEYNMAN INTEGRALS}

In this section, we calculate all the two-dimensional integrals needed for the matrix element. The only integral, that must be solved in terms of analytic functions, is the one with a two-term denominator, the others can then be reduced to this one.

\section{The Two-Term Denominator}

This is the integral of the form

$$
\begin{aligned}
I\left(k_{2}, m_{1}^{2}, m_{2}^{2}\right): & =\int d^{2} q \\
& \times\left[\left(q^{2}+m_{1}^{2}\right)\left(\left(q+k_{2}\right)^{2}+m_{2}^{2}\right)\right]^{-1} .
\end{aligned}
$$

Introducing standard Feynman parameters, we find

$$
\begin{aligned}
I & =\int_{0}^{1} d x \int d^{2} q \\
& \times\left[\left(q^{2}+m_{1}^{2}\right) x+\left(\left(q+k_{2}\right)^{2}+m_{2}^{2}\right)(1-x)\right]^{-2} .
\end{aligned}
$$

The term in bracket can now be put into the form 


$$
\left(q+k_{2}(1-x)\right)^{2}+k_{2}^{2} x(1-x)+m_{1}^{2} x+m_{2}^{2}(1-x)
$$

giving for the integral

$$
\begin{aligned}
I & =\int_{0}^{1} d x \int d^{2} q\left[\left(q+k_{2}(1-x)\right)^{2}+k_{2}^{2} x(1-x)+m_{1}^{2} x+m_{2}^{2}(1-x)\right]^{-2} \\
& =\pi \int_{0}^{1} d x\left[k_{2}^{2} x(1-x)+m_{1}^{2} x+m_{2}^{2}(1-x)\right]^{-1} .
\end{aligned}
$$

The evaluation of the last integral is straightforward and we get

$$
I\left(k_{2}, m_{1}^{2}, m_{2}^{2}\right)=\pi \ln \left(\frac{\left(k_{2}^{2}+m_{1}^{2}+m_{2}^{2}+s\right)^{2}}{4 m_{1}^{2} m_{2}^{2}}\right) /\left(\left(m_{1}^{2}-m_{2}^{2}\right)^{2}+k_{2}^{2}\left(k_{2}^{2}+2 m_{1}^{2}+2 m_{2}^{2}\right)\right)^{1 / 2}
$$

with

$$
\text { . : }=\left(\left(k_{2}^{2}+m_{2}^{2}+m_{1}^{2}\right)^{2}-4 m_{1}^{2} m_{2}^{2}\right)^{1 / 2} \text {. }
$$

Note, that the final result only depends on the values of $k_{2}^{2}, m_{1}^{2}$ and $m_{2}^{2}$.

\section{The Three-Term Denominator}

This is the integral

$$
\begin{aligned}
& I^{S}\left(k_{2}, k_{3}, m_{1}^{2}, m_{2}^{2}, m_{3}^{2}\right):=\int d^{2} q \\
& \times\left[\left(q^{2}+m_{1}^{2}\right)\left(\left(q+k_{2}\right)^{2}+m_{2}^{2}\right)\left(\left(q+k_{3}\right)^{2}+m_{3}^{2}\right)\right]^{-1} .
\end{aligned}
$$

In principal, an analytic form can be found directly doing the integration over two suxiliary variables, using the trick described by t'Hooft and Veltman [20]. This calculation is rather tedious and it is easier to use a formula by Vermaseren and van Neerven [21] for the reduction of a $(N+1)$-term integral in $N$ Dimension into $N+1 N$-term integrals.

Their formula (20) in our notation is

$$
\begin{aligned}
& I^{S}\left(k_{2}, k_{3}, m_{1}^{2}, m_{2}^{2}, m_{3}^{2}\right)=\left(\left(r_{2} k_{3}-r_{3} k_{2}\right)^{2}+4\left(k_{2}^{2} k_{3}^{2}-\left(k_{2} k_{3}\right)^{2}\right) m_{1}^{2}\right)^{-1} \\
& \times\left(\left(2\left(k_{2}^{2} k_{3}^{2}-\left(k_{2} k_{3}\right)^{2}\right)-r_{2} k_{3}^{2}-r_{3} k_{2}^{2}+\left(r_{2}+r_{3}\right) k_{2} k_{3}\right) I\left(k_{3}-k_{2}, m_{2}^{2}, m_{3}^{2}\right)\right. \\
& \left.+\left(r_{2} k_{3}^{2}-r_{3} k_{2} k_{3}\right) I\left(k_{3}, m_{1}^{2}, m_{3}^{2}\right)+\left(r_{3} k_{2}^{2}-r_{2} k_{2} k_{3}\right) I\left(k_{2}, m_{1}^{2}, m_{2}^{2}\right)\right),
\end{aligned}
$$

with

$$
\begin{aligned}
& r_{2}=m_{2}^{2}+k_{2}^{2}-m_{1}^{2} \\
& r_{3}=m_{3}^{2}+k_{3}^{2}-m_{1}^{2} .
\end{aligned}
$$

Note again, that the final result only depends on $m_{1}^{2}, m_{2}^{2}, m_{3}^{2}, k_{2}^{2}, k_{3}^{2}$ and $k_{2} k_{3}$. 


\section{The Tensor Integral}

The only tensor integral, which appears in our calculation is the one with one momentum in the numerator. Generally, all tensor integrals can be reduced to the acalar integrals. Here we use the method, which is for example described in t'Hooft and Veltman [20].

The standard form of the integral is:

$$
I^{i}\left(k_{2}, k_{3}, m_{1}^{2}, m_{2}^{2}, m_{3}^{2}\right)=\int d^{2} q q^{i}\left[\left(q^{2}+m_{1}^{2}\right)\left(\left(q+k_{2}\right)^{2}+m_{2}^{2}\right)\left(\left(q+k_{3}\right)^{2}+m_{3}^{2}\right)\right]^{-1} \text {. }
$$

As $k_{2}$ and $k_{3}$ are the only variables in the integral with a vector character, the integral can be split into

$$
I^{i}=k_{2}^{i} I^{2}+k_{3}^{i} I^{3}
$$

To find expreasions for $I^{2}$ and $I^{3}$, we multiply with $k_{2}$ and $k_{3}$ :

$$
\begin{gathered}
\left(k_{2} I\right)=k_{2}^{2} I^{2}+k_{2} k_{3} I^{3} \\
\left(k_{3} I\right)=k_{2} k_{3} I^{2}+k_{3}^{2} I^{2} .
\end{gathered}
$$

Salving for $I^{2}$ and $I^{3}$, we get

$$
\begin{aligned}
& I^{2}=\left[k_{2}^{2} k_{3}^{2}-\left(k_{2} k_{3}\right)^{2}\right]^{-1}\left(k_{3}^{2}\left(k_{2} I\right)-k_{2} k_{3}\left(k_{3} I\right)\right) \\
& I^{3}=\left[k_{2}^{2} k_{3}^{2}-\left(k_{2} k_{3}\right)^{2}\right]^{-1}\left(-k_{2} k_{3}\left(k_{2} I\right)+k_{2}^{2}\left(k_{3} I\right)\right) .
\end{aligned}
$$

Now with the help of

$$
\begin{aligned}
& q k_{2}=\frac{1}{2}\left(\left(\left(q+k_{2}\right)^{2}+m_{2}^{2}\right)-\left(q^{2}+m_{1}^{2}\right)-r_{2}\right) \\
& q k_{3}=\frac{1}{2}\left(\left(\left(q+k_{3}\right)^{2}+m_{3}^{2}\right)-\left(q^{2}+m_{1}^{2}\right)-r_{3}\right)
\end{aligned}
$$

we get

$$
\begin{aligned}
\left(k_{2} I\right) & =\int d^{2} q\left(k_{2} q\right)\left[\left(q^{2}+m_{1}^{2}\right)\left(\left(q+k_{2}\right)^{2}+m_{2}^{2}\right)\left(\left(q+k_{3}\right)^{2}+m_{3}^{2}\right)\right]^{-1} \\
& =\frac{1}{2} I\left(k_{3}, m_{1}^{2}, m_{2}^{2}\right)-\frac{1}{2} I\left(k_{3}-k_{2}, m_{2}^{2}, m_{3}^{2}\right)-\frac{1}{2} r_{2} I^{S}\left(k_{2}, k_{3}, m_{1}^{2}, m_{2}^{2}, m_{3}^{2}\right)
\end{aligned}
$$

and aimilar for

$$
\begin{aligned}
\left(k_{3} I\right) & =\int d^{2} q\left(k_{3} q\right)\left[\left(q^{2}+m_{1}^{2}\right)\left(\left(q+k_{2}\right)^{2}+m_{2}^{2}\right)\left(\left(q+k_{3}\right)^{2}+m_{3}^{2}\right)\right]^{-1} \\
& =\frac{1}{2} I\left(k_{2}, m_{1}^{2}, m_{2}^{2}\right)-\frac{1}{2} I\left(k_{3}-k_{2}, m_{2}^{2}, m_{3}^{2}\right)-\frac{1}{2} r_{3} I^{S}\left(k_{2}, k_{3}, m_{1}^{2}, m_{2}^{2}, m_{3}^{2}\right)
\end{aligned}
$$

and finally for $I^{2}$ and $I^{3}$

$$
\begin{aligned}
I^{2} & =\left[2\left(k_{2}^{2} k_{3}^{2}-\left(k_{2} k_{3}\right)^{2}\right)\right]^{-1}\left(\left(k_{2} k_{3}-k_{3}^{2}\right) I\left(k_{3}-k_{2}, m_{2}^{2}, m_{3}^{2}\right)+k_{3}^{2} I\left(k_{3}, m_{1}^{2}, m_{3}^{2}\right)\right. \\
& \left.-k_{2} k_{3} I\left(k_{2}, m_{1}^{2}, m_{2}^{2}\right)+\left(r_{3} k_{2} k_{3}-r_{2} k_{3}^{2}\right) I^{S}\left(k_{2}, k_{3}, m_{1}^{2}, m_{2}^{2}, m_{3}^{2}\right)\right)
\end{aligned}
$$




$$
\begin{aligned}
I^{3}= & {\left[2\left(k_{2}^{2} k_{3}^{2}-\left(k_{2} k_{3}\right)^{2}\right)\right]^{-1}\left(\left(k_{2} k_{3}-k_{2}^{2}\right) I\left(k_{3}-k_{2}, m_{2}^{2}, m_{3}^{2}\right)+k_{2}^{2} I\left(k_{2}, m_{1}^{2}, m_{2}^{2}\right)\right.} \\
& \left.-k_{2} k_{3} I\left(k_{3}, m_{1}^{2}, m_{3}^{2}\right)+\left(r_{2} k_{2} k_{3}-r_{3} k_{2}^{2}\right) I^{S}\left(k_{2}, k_{3}, m_{1}^{2}, m_{2}^{2}, m_{3}^{2}\right)\right) .
\end{aligned}
$$

\section{Teating the Formula}

The following two properties of the integrals have been used to test the results: First, the symmetry of the formulas with reapect to the exchange of $N_{1}, N_{2}$ and $N_{3}$ has been teated. Second, a teat can be done, based on the following mathematical property [20]:

Starting from

$$
\frac{1}{\left(q^{2}+m_{1}^{2}\right)\left(\left(q+k_{2}\right)^{2}+m_{2}^{2}\right)}
$$

we multiply numerator and denominator with

$$
\begin{aligned}
& (1-\alpha)\left(q^{2}+m_{1}^{2}\right)+\alpha\left(\left(q+k_{2}\right)^{2}+m_{2}^{2}\right) \\
& =\left(q+\alpha k_{2}\right)^{2}+\alpha m_{2}^{2}+(1-\alpha) m_{1}^{2}+\alpha(1-\alpha) k_{2}^{2} \\
& =:(q+K)^{2}+M^{2}
\end{aligned}
$$

where $\alpha$ is an arbitrary real parameter. With this we find

$$
\begin{aligned}
& \frac{1}{\left(q^{2}+m_{1}^{2}\right)\left(\left(q+k_{2}\right)^{2}+m_{2}^{2}\right)} \\
& =\frac{1-\alpha}{\left(\left(q+k_{2}\right)^{2}+m_{2}^{2}\right)\left((q+K)^{2}+M^{2}\right)} \\
& +\frac{\alpha}{\left(q^{2}+m_{1}^{2}\right)\left((q+K)^{2}+M^{2}\right)}
\end{aligned}
$$

The identity between the resulting three integrals can then be verified.

\section{APPENDIX B; DISCUSSION OF THE FORM FACTORS}

Normally a Gaussian form factor, or that of a homogeneous charged sphere are used. They are given by (note that in our metric $q^{2}<0$ ):

$$
\begin{aligned}
& F_{\text {Gawoo }}\left(q^{2}\right)=\exp \left(-\frac{\left|q^{2}\right|}{2 Q_{0}^{2}}\right) \\
& F_{\text {h.c.s. }}\left(q^{2}\right)=\frac{3 j_{1}\left(\sqrt{\left|q^{2}\right|} R_{0}\right)}{\sqrt{\left|q^{2}\right|} R_{0}}
\end{aligned}
$$

with $Q_{0}=60 \mathrm{MeV}$ and $R_{0}=1.2 \mathrm{fm} A^{1 / 3} \approx 7 \mathrm{fm}$. 
We are using two different form factors, which have the advantage, that our matrix element can be expressed analytically with them. One is the dipole form factor

$$
F_{\text {dipole }}\left(q^{2}\right)=\frac{\Lambda^{2}}{\Lambda^{2}-q^{2}}
$$

which is the form factor of a Yukawa charge distribution

$$
\rho(r)=\frac{\Lambda^{2}}{4 \pi} \frac{\exp (-\Lambda r)}{r} .
$$

$\Lambda$ has been determined 20 , that the root mean square of the electric radius is equal to the experimental value

$$
\sqrt{\left\langle r^{2}\right\rangle}=\sqrt{\frac{6}{\Lambda^{2}}}=1 \mathrm{fm} A^{1 / 3}
$$

giving a $\Lambda$ of about $83 \mathrm{MeV}$. This value has been used throughout the calculations, even though it varies alightly with $A$ for example for $P b$ to $A u$.

A comparien of the different form factors can be found in Fig. 16. The dieadvantage of the dipole form factor is, that it decreases too alowly and overestimates the ros value, if $q$ is larger than about $50 \mathrm{MeV}$. To ree, if our result depends on the exact form of the form factor, we use a eccond one, which has a better behavior for large $q$. It is as sum of two dipole form factors:

$$
F_{\text {dowbe }}\left(q^{2}\right)=c_{1} \frac{\Lambda_{1}^{2}}{\Lambda_{1}^{2}-q^{2}}+c_{2} \frac{\Lambda_{2}^{2}}{\Lambda_{2}^{2}-q^{2}}
$$

Therefore we call it a "double dipole form factor". With $F(0)=1$, we have $c_{1}+c_{2}=1$. The behavior of this form factor for large values of $|q|$ is

$$
F\left(q^{2}\right) \sim \frac{\Lambda_{1}^{2} \Lambda_{2}^{2}-\left(c_{1} \Lambda_{1}^{2}+c_{2} \Lambda_{2}^{2}\right) q^{2}}{q^{4}}
$$

For that the form factor vanishes faster than $1 / q^{2}$, the coefficient in front of $q^{2}$ has to be zero, therefore $\Lambda_{2}^{2}$ has to be chosen as

$$
\Lambda_{2}^{2}=-\frac{c_{1}}{c_{2}} \Lambda_{1}^{2} \text {. }
$$

As we do not want to have a singularity in the form factor, we have to choose $\Lambda_{2}^{2}>0$, that is $\left.c_{1}\right\rangle$ 1. Finally, we want, that $\left\langle r^{2}\right\rangle$ is again the same as the experimental one. As the density corresponding to this form factor is

$$
\rho(r)=c_{1} \frac{\Lambda_{1}^{2}}{4 \pi} \frac{\exp \left(-\Lambda_{1} r\right)}{r}+c_{2} \frac{\Lambda_{2}^{2}}{4 \pi} \frac{\exp \left(-\Lambda_{2} r\right)}{r},
$$

- again a sum of two Yukawa charge densities $-\left\langle r^{2}\right\rangle$ is

$$
\left\langle r^{2}\right\rangle=6\left(\frac{c_{1}}{\Lambda_{1}^{2}}+\frac{c_{2}}{\Lambda_{2}^{2}}\right)
$$


Therefore

$$
\Lambda_{1}^{2}=\frac{6}{\left\langle r^{2}\right\rangle}\left(2-\frac{1}{c_{1}}\right)
$$

and $c_{1}$ is the only parameter, which can be varied. For $c_{1}=1$, we have the normal dipole form factor, for $c_{1} \rightarrow \infty$ it decreases faster than it. In Fig. 16 we have plotted $F_{\text {double }}(q)$ for $c_{1}=10$, a value, that we have also used throughout the calculation, because the cancellations between the two dipole terms are not too large.

The reason, why theae form factors can be treated analytically, is due to the fact, that with the dipole form factor, we can write

$$
\frac{F_{\text {dipole }}\left(q^{2}\right)}{q^{2}}=\frac{1}{q^{2}} \frac{\Lambda^{2}}{\Lambda^{2}-q^{2}}=\frac{1}{q^{2}}+\frac{1}{\Lambda^{2}-q^{2}},
$$

having a term of the form $1 /\left(q^{2}+m^{2}\right)$ again. Therefore our momentum integrals can be written as sum and differences of the standard Feynman integrals. 


\section{REFERENCES}

[1] G. Baur, Phys. Rev. A42, 5736 (1990)

[2] M.J. Rhoades-Brown, J. Weneser, Phys. Rev. A44, 330 (1991)

[3] C. Best, W. Greiner, G. Soff, Phys. Rev. A46, 261 (1992)

[4] K.F. Weizsäcker, Z. Phys. 88, 612 (1934)

[5] E.J. Williams, Phys. Rev. 45, 729 (1934)

[6] L.D. Landau, E.M. Lifshitz, Phys. Z. Sowjet. 6, 244 (1934)

[7] A.I. Achieser, W.B. Beresterki, Quanten-Elektrodymamik (B.G. Teubner Verlag, Leiprig, 1962)

[8] C. Bottcher, M.R. Strayer, Phys. Rev. D39, 1330 (1989)

[9] FORM is an algebraical calculation program by J.A.M. Vermaseren, the free veraion 1.0 can be found e.g. at FTP.NIKHEF.NL

[10] C.A. Bertulani, G. Baur, Phys. Rep. 163, 299 (1988)

[11] V.M. Budnev, I.F. Gineburg, G.V. Meledin, V.G. Serbo, Phys. Rep. 15, 181 (1975)

[12] G. Baur and L.G. Ferreira Filho, Phys. Lett. B234, 30 (1991)

[13] G. Baux, Z. Phys. Cs4, 419 (1992)

[14] M. Vidorić, M. Greiner, Ch. Best, G. Soff, Phys. Rev. C47, 2308 (1993)

[15] G.P. Lepage, J. Comp. Phys. 27, 192 (1978)

[16] G.P. Lepage, CLNS-80/447 (1980)

[17] G. Baur, N. Baron, Nucl. Phys. A 561 (1993) 629

[18] N. Baron, G. Baur, Z. Phys. C (in press)

[19] H. Bethe, W. Heitler, Proc. Roy. Soc. 146, 83 (1934)

[20] G.'Hooft, M. Veltmen, Nucl. Phys. B 153, 265 (1979)

[21] W.L. van Neerven, J.A.M. Vermaseren, Phys. Lett. B137, 241 (1984) 


\section{FIGURES}

FIG. 1. General form of the second order diagram for pair creation in an external field.

FIG. 2. The two diagrams contributing to the pair creation in the heavy-ion collisions, where (1) and (2) denote the interaction with the field of ion 1 and 2 respectively.

FIG. 3. $P_{\text {lolal }} /(Z \alpha)^{4}$ for $b=0$ a a function of $\Lambda$ for $\gamma=100$ (lower curve) and 3400 (upper curve). The logarithmic increase shows, that a form factor is needed, as the result for a point charge neems to diverge.

FIG. 4. $P_{\text {total }} /(Z a)^{4}$ for $b=0$ as a function of $\gamma$ for the creation of an alectron-ponitron pair. The solid line is the calculation for a realistic dipale form factor, the dotted line for a realistic double dipole form factor (see App. B). The dashed lines are EPA results for impact parameters $b=0.5 \lambda_{c}, b=1.0 \lambda_{c}$ and $b=1.5 \lambda_{c}$ (from left to right) respectively.

FIG. 6. $P_{\text {bad }} /(Z \alpha)^{4}$ for $b=0$ a a function of $\gamma$ for the creation of an eloctron- (colid), $\mu$ (dotted), and r-pair (dached). Realts of the calculation with a dipole form factor.

FIG. 6. A comparison of our calculation (solid) and DEPA (dotted) for $\gamma=100$ as a function of $\Lambda_{1}$ of one ion. $\Lambda_{2}$ of the other ion has been kept fixed at $\Lambda_{2}=0.1,1,10,100 \mathrm{MeV}$ (from bottom to top).

FIG. 7. $P(E) /(Z \alpha)^{4}$ as a function of the energy of the positron. Results for $\gamma=10,100,3400$ (from bottom to top) are shown, solid lines are the results using the realiatic dipole form factor, dotted lines using the realistic double dipole form factor.

FIG. 8. $P(\theta) /(Z \alpha)^{4} a$ a function of the angle $\theta$ of $p_{+}$with the s-axis. $\gamma$ and form factors as in Fig. 7.

FIG. 9. $P(M) /(Z \alpha)^{4}$ as a function of the invariant mass $M . \gamma$ and form factors as in Fig. 7 .

FIG. 10. $P(Y) /(Z \alpha)^{4}$ as a function of the rapidity of the pair. $\gamma$ and form factors as in Fig. 7 .

FIG. 11. $P\left(P_{\perp}\right) /(Z \alpha)^{4}$ as a function of the transverse momentum $P_{\perp}$ of the pair. $\gamma$ and form factors as in Fig. 7 .

FIG. 12. $P(M) /(Z \alpha)^{4}$ is shown a a function of $M$ for pairs with large invariant mase $M$. Comparieon for $\gamma=100,3400$ of our calculation (solid line) with the DEPA (dotted line), using a realistic dipole form factor in both cases. 
FIG. 13. $P(M, Y=0) /(Z \alpha)^{4}$ a a function of $M$. Comparison for $\gamma=100$ (lower curve) and 3400 (upper curve) of our calculation (solid line) with the DEPA (dotted line). Results for the realistic double dipole form factor are shown.

FIG. 14. $P(M, Y=0, \Omega) /(Z \alpha)^{4}$ as a function of the angle $\theta$ between $p_{+}$and the s-axis. Compared are the results of our calculation (solid line) and DEPA (dotted line). $\gamma=3400$, $M=500 \mathrm{MeV}$ (upper curve), $3500 \mathrm{MeV}$ (lower curve). Results for the realistic double dipole form factor are ahown.

FIG. 15. Same a Fig.14, but now $P\left(M, Y=0, \Omega_{\Delta}\right) /(Z \alpha)^{4}$ as a function of the angle $\theta_{\Delta}$ between $\left(p_{+}-p_{-}\right)$and the s-aris.

FIG. 16. A comparison of the different form factors. The dashed line is the Gauss form factor, the dashed-dotted line the form factor of a homogenoously charged sphere. The colid line is the imple dipole form factor, the dotted line the double dipole form factor with parameter $c_{1}=10$. 
TABLES

\begin{tabular}{llll}
\hline \hline & $\gamma$ & Ion & $P_{\text {total }}$ \\
\hline AGS & 2.35 & $\mathrm{Au}$ & $\mathbf{0 . 0 6}$ \\
CERN SPS & 10 & $\mathrm{~Pb}$ & 0.63 \\
RHIC & 100 & $\mathrm{Au}$ & 1.6 \\
LHC & 3400 & $\mathrm{~Pb}$ & 3.9 \\
SSC & 8000 & $\mathrm{~Pb}$ & 4.4 \\
\hline \hline
\end{tabular}

TABLE I. Predicted values of $P_{\text {cotal }}$ for different accelerator parameters 



$$
\frac{T}{L E}
$$


. 


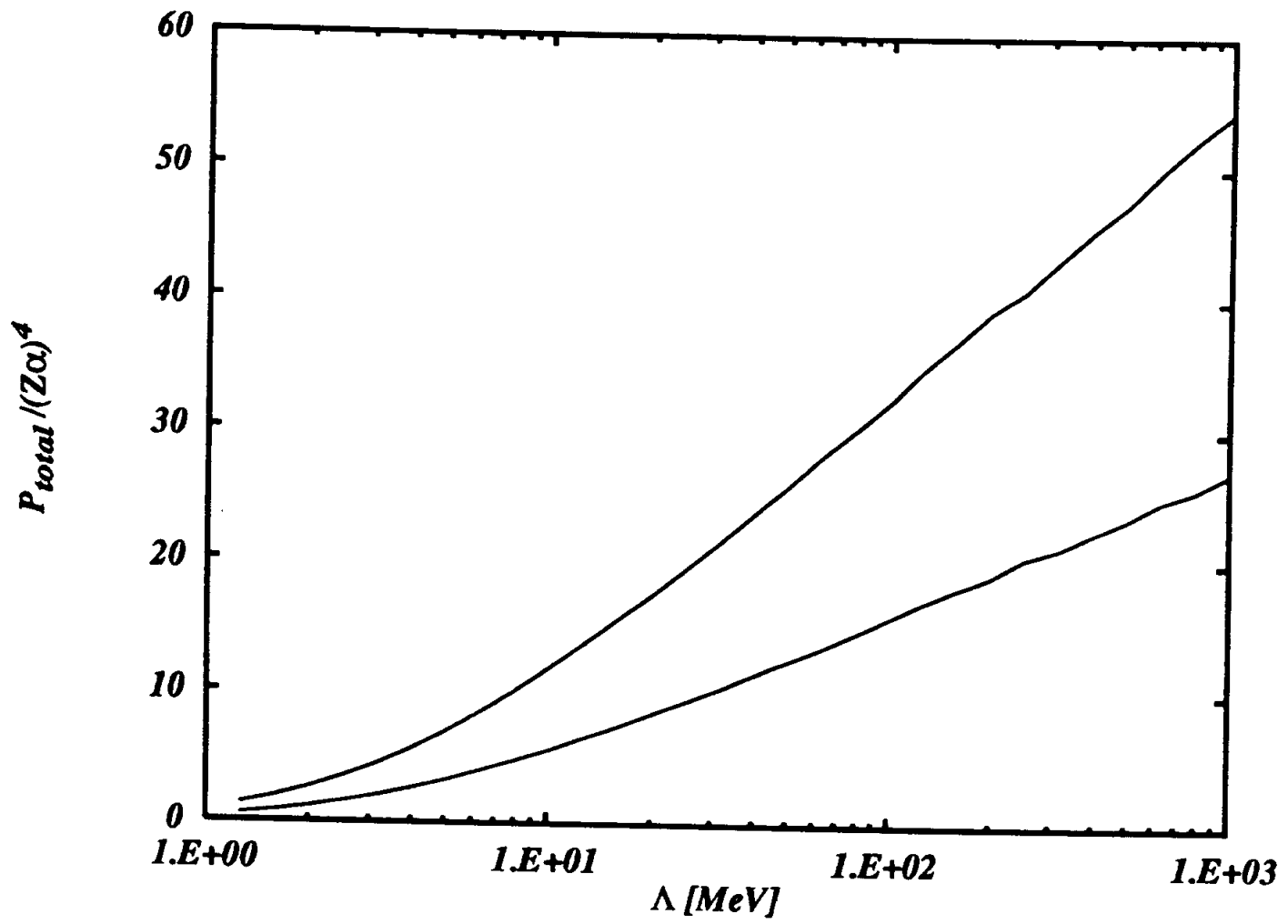

FIG. 3

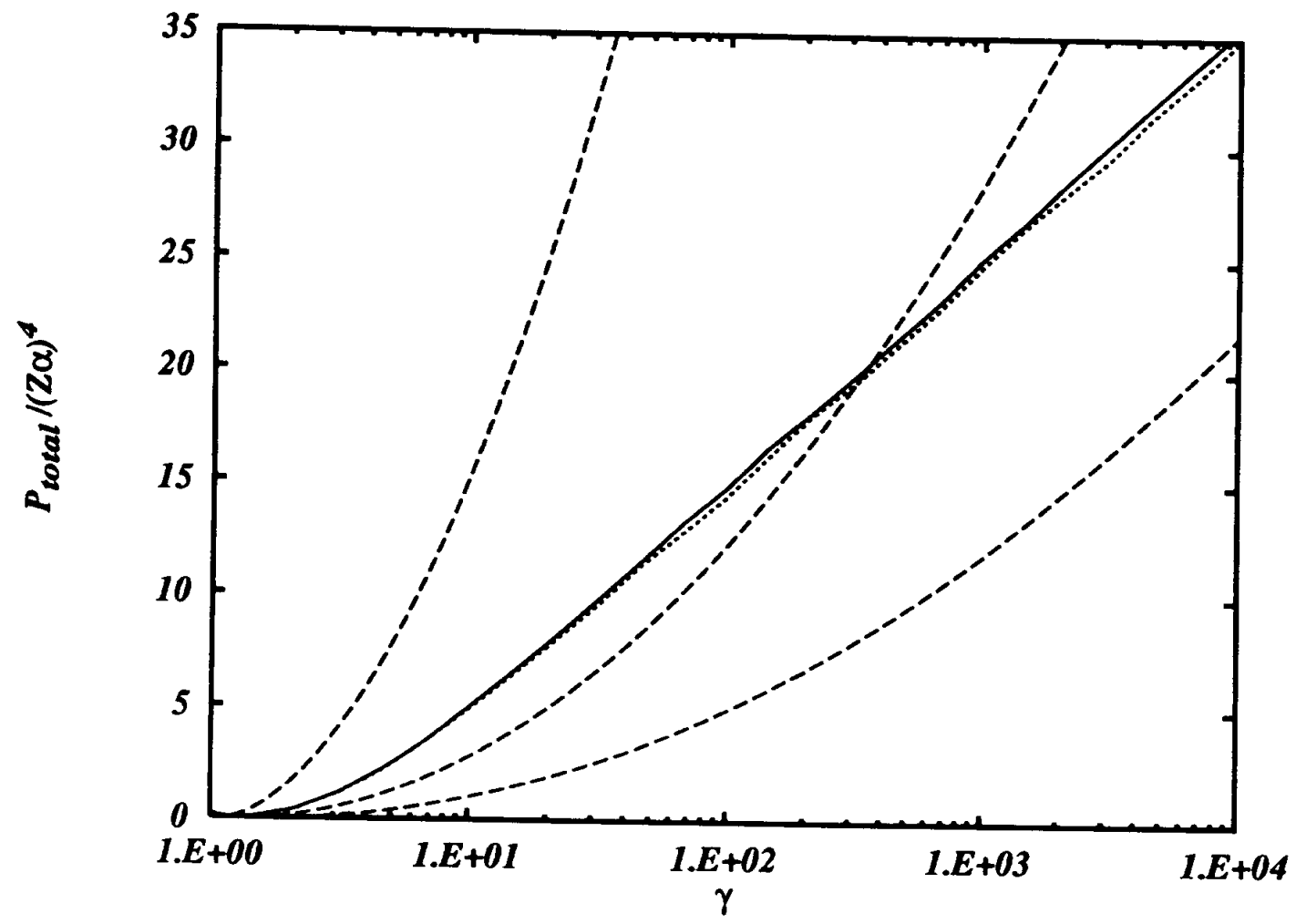

FIG. 4 


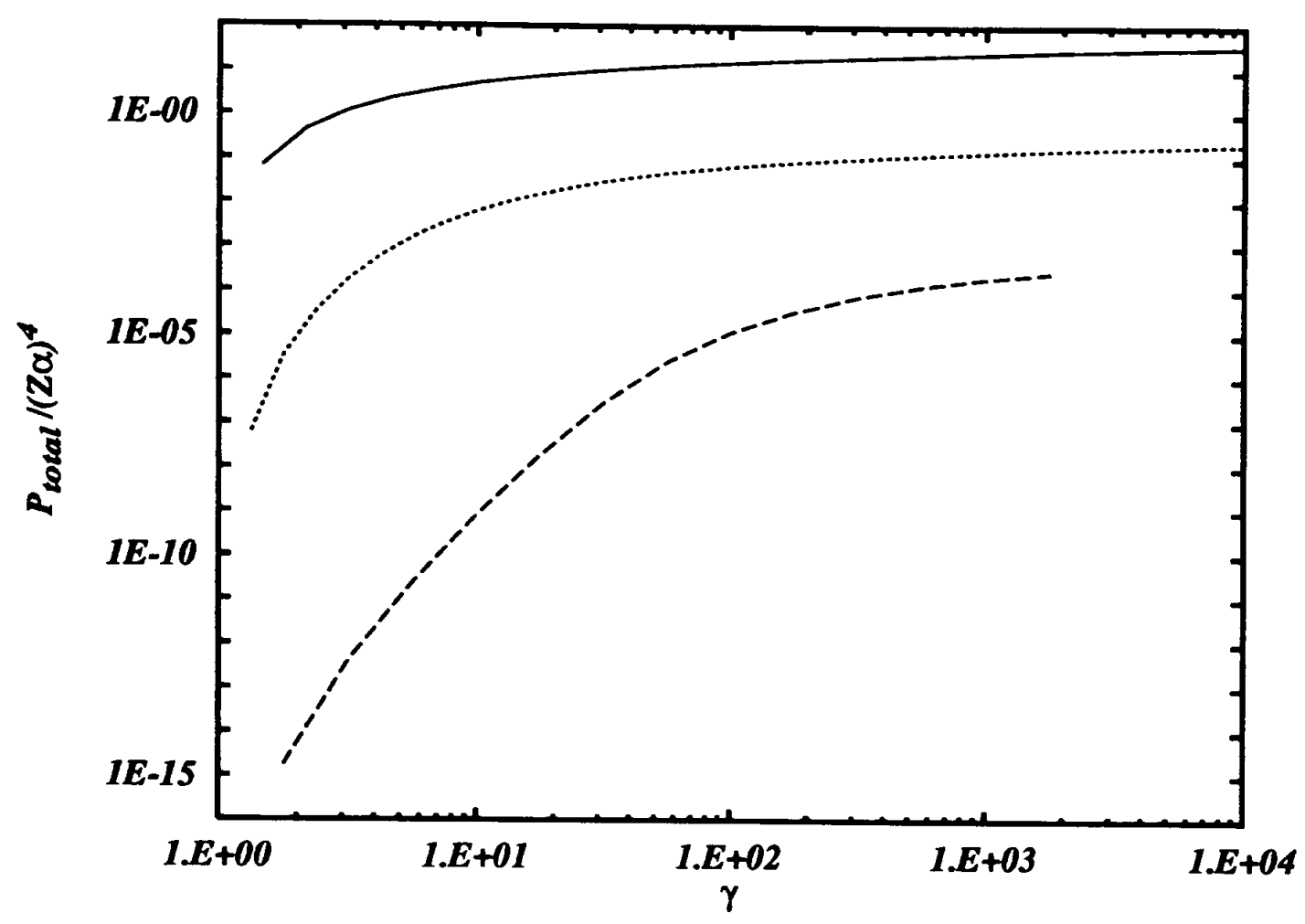

FIG. 5

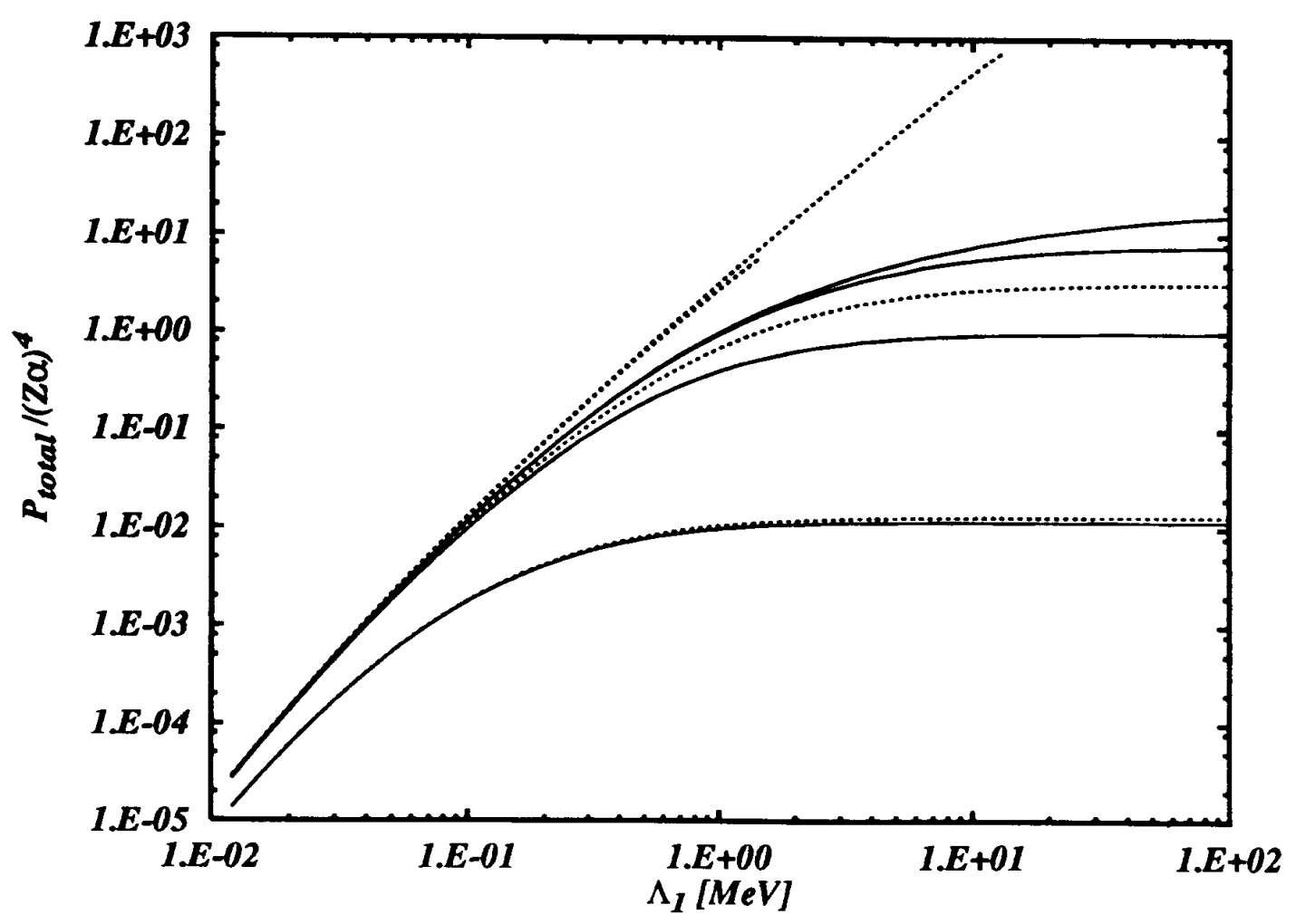

FIG. 6 



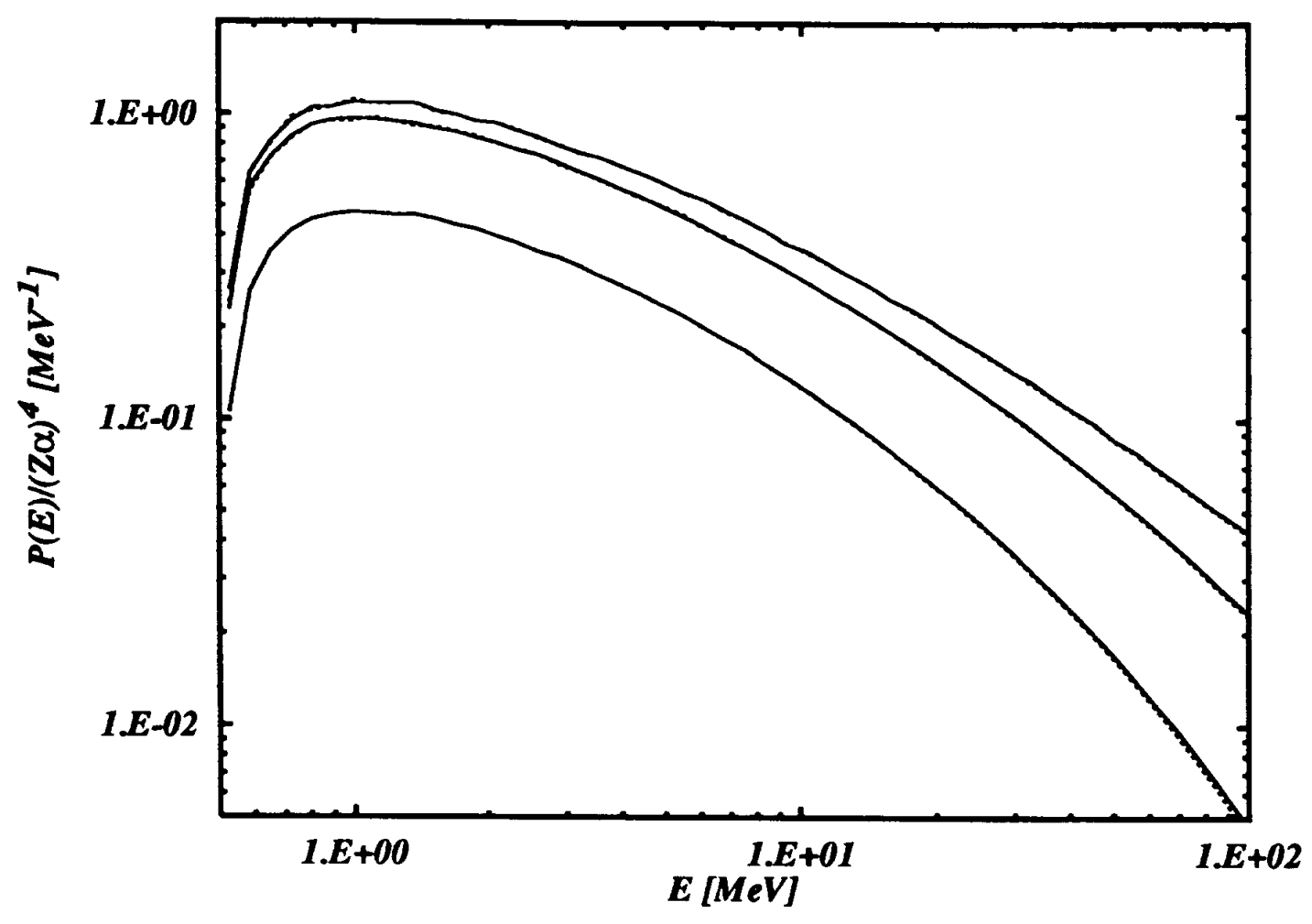

FIG. 7

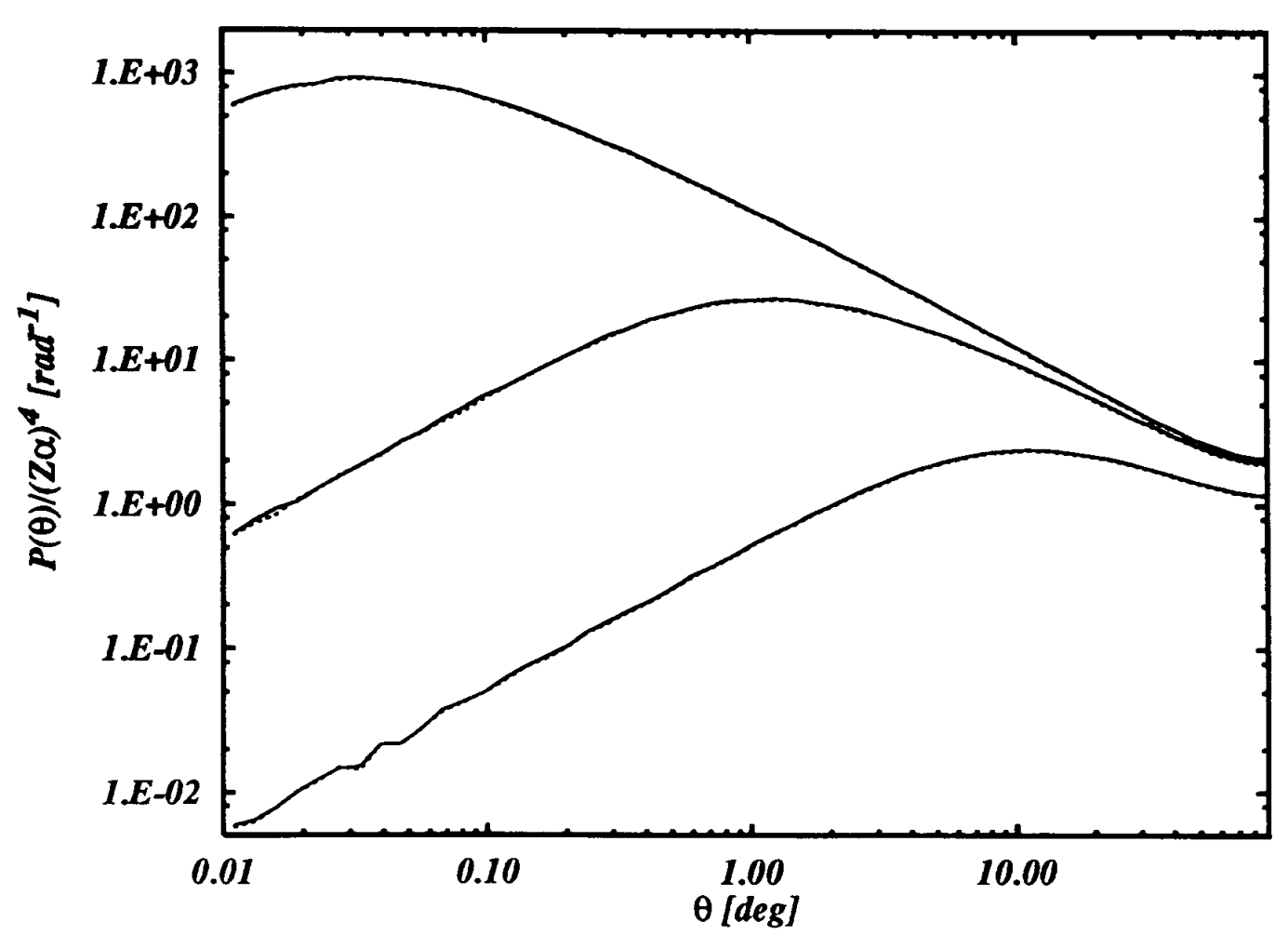

FIG. 8 



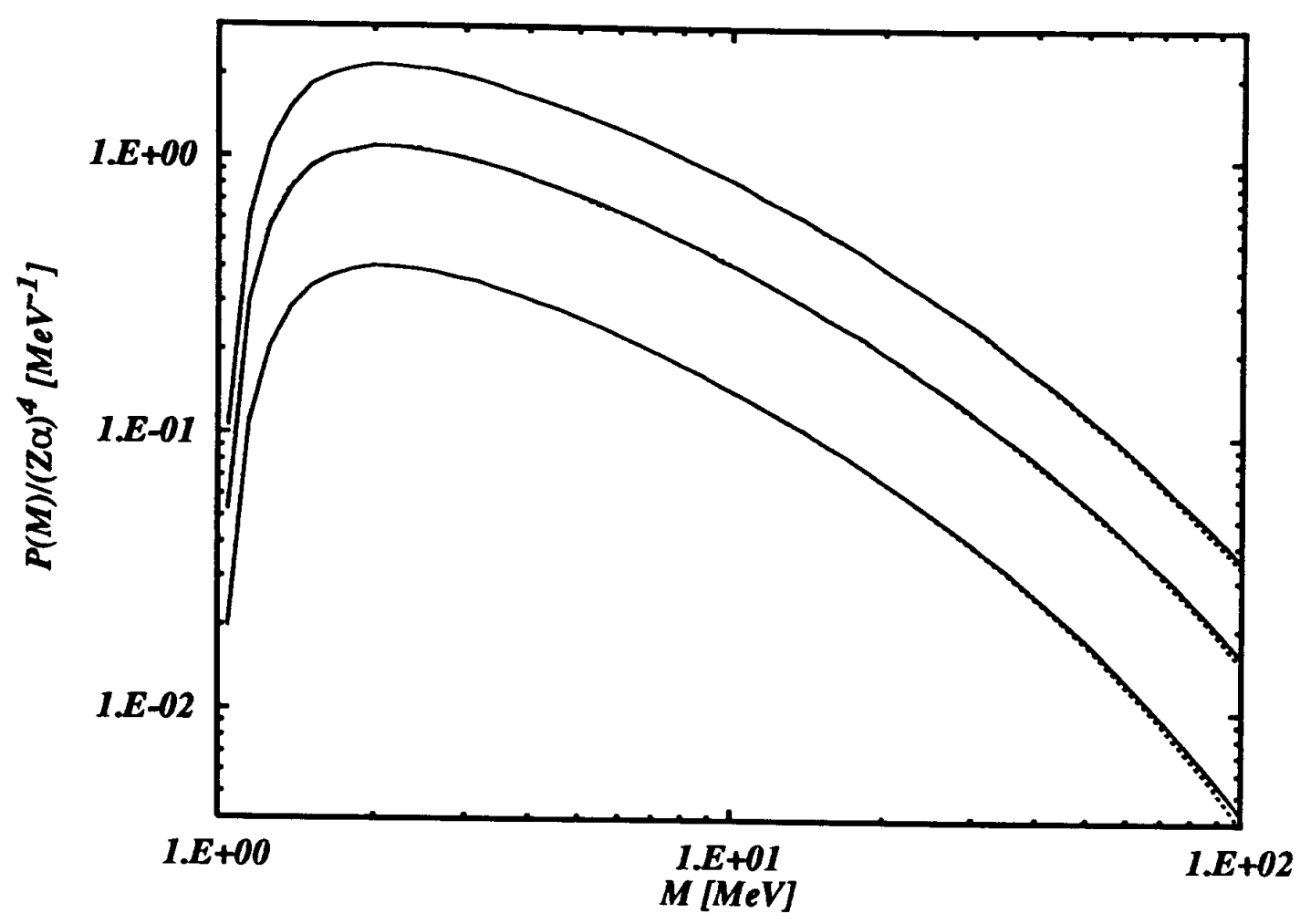

FIG. 9

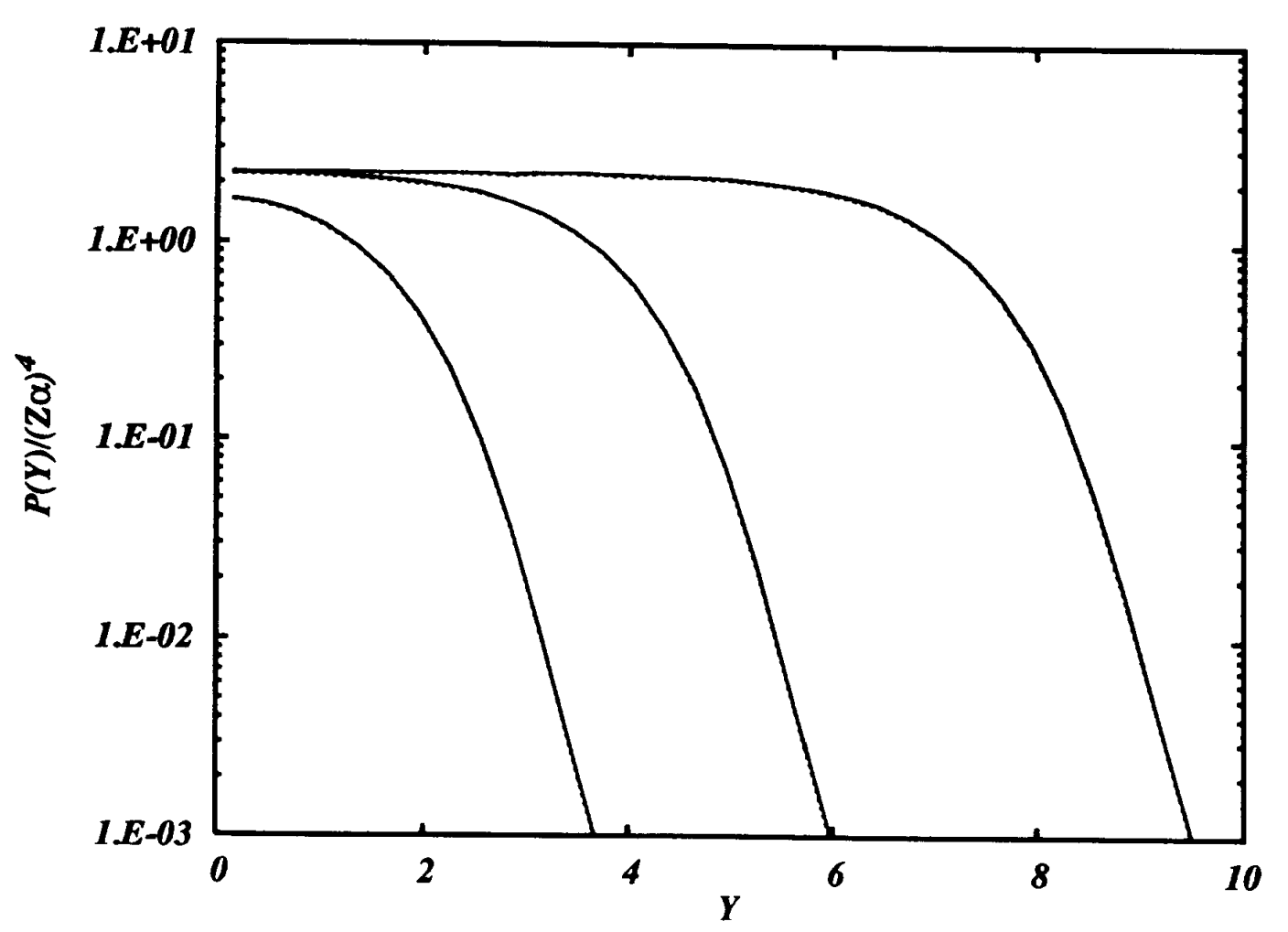

FIG. 10 


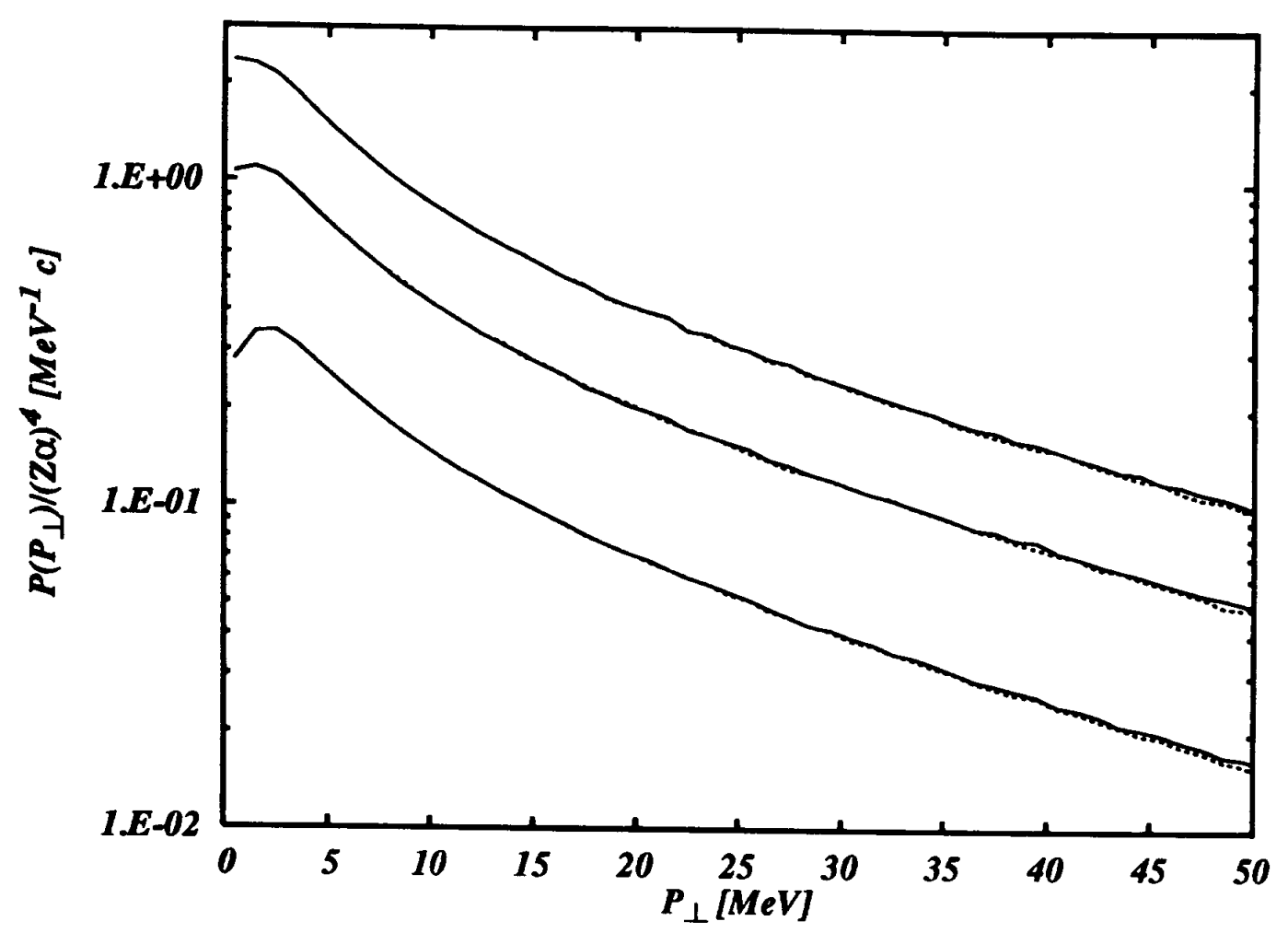

FIG. 11

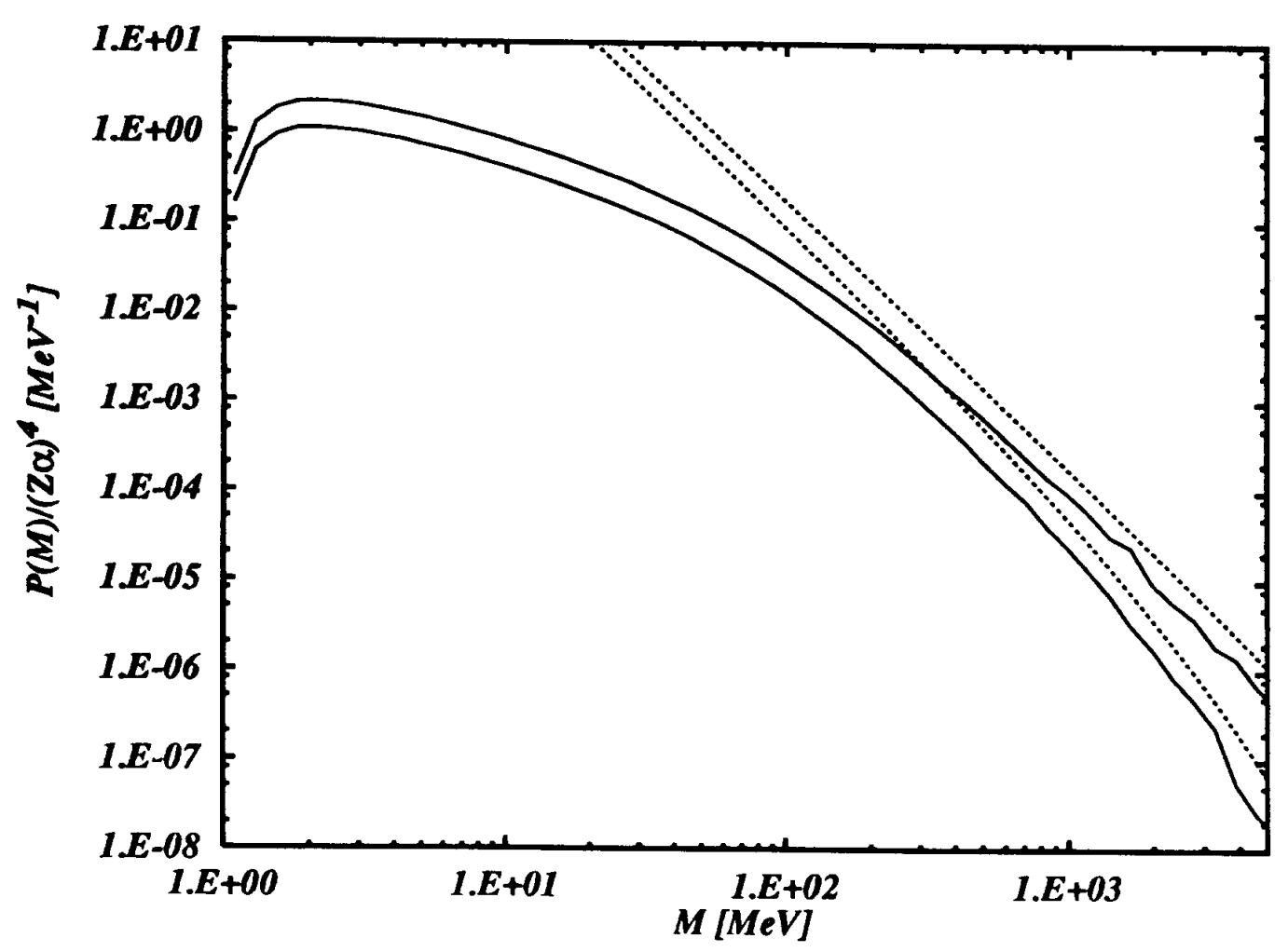

FIG. 12 



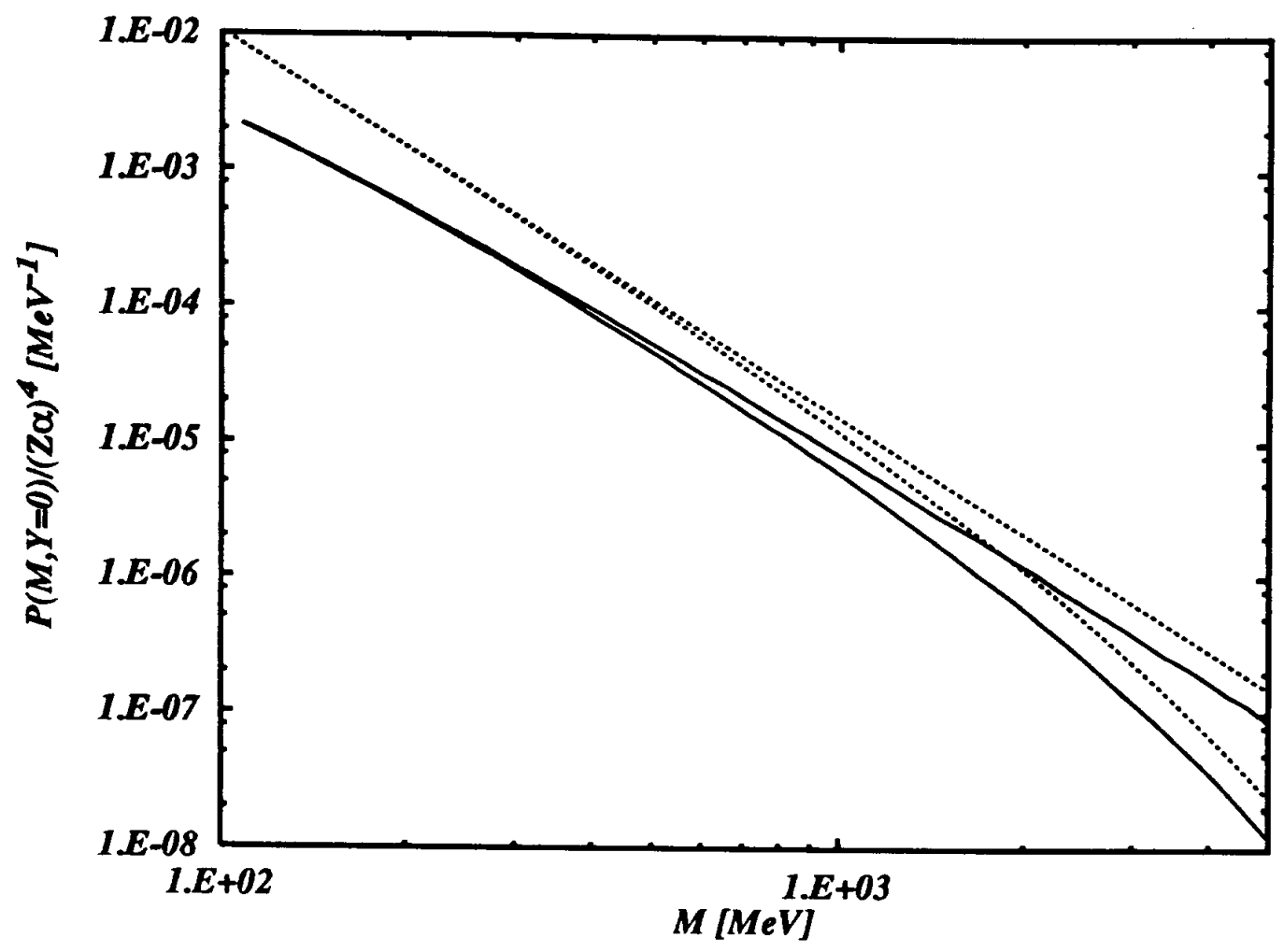

FIG. 13

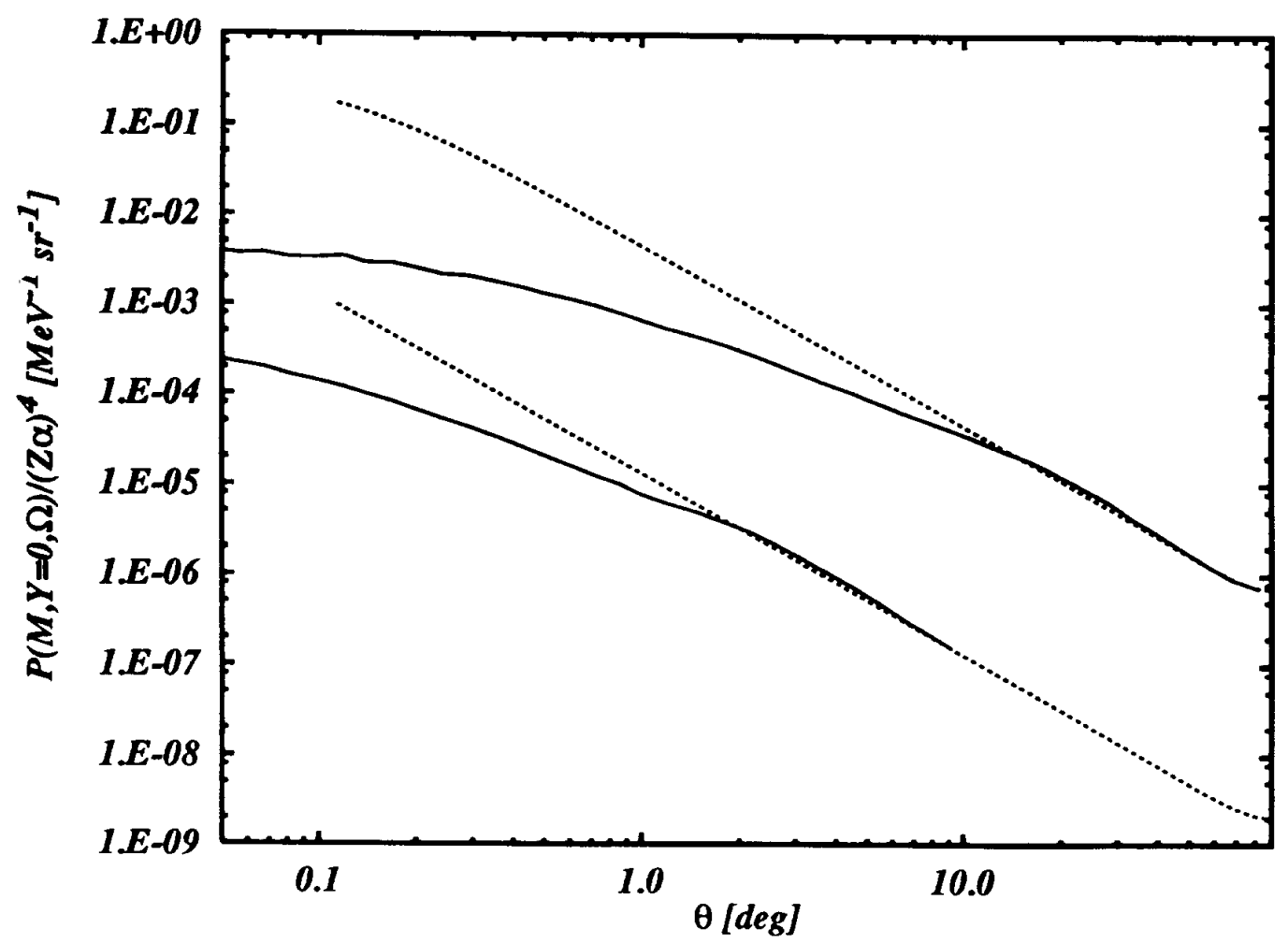

FIG. 14 


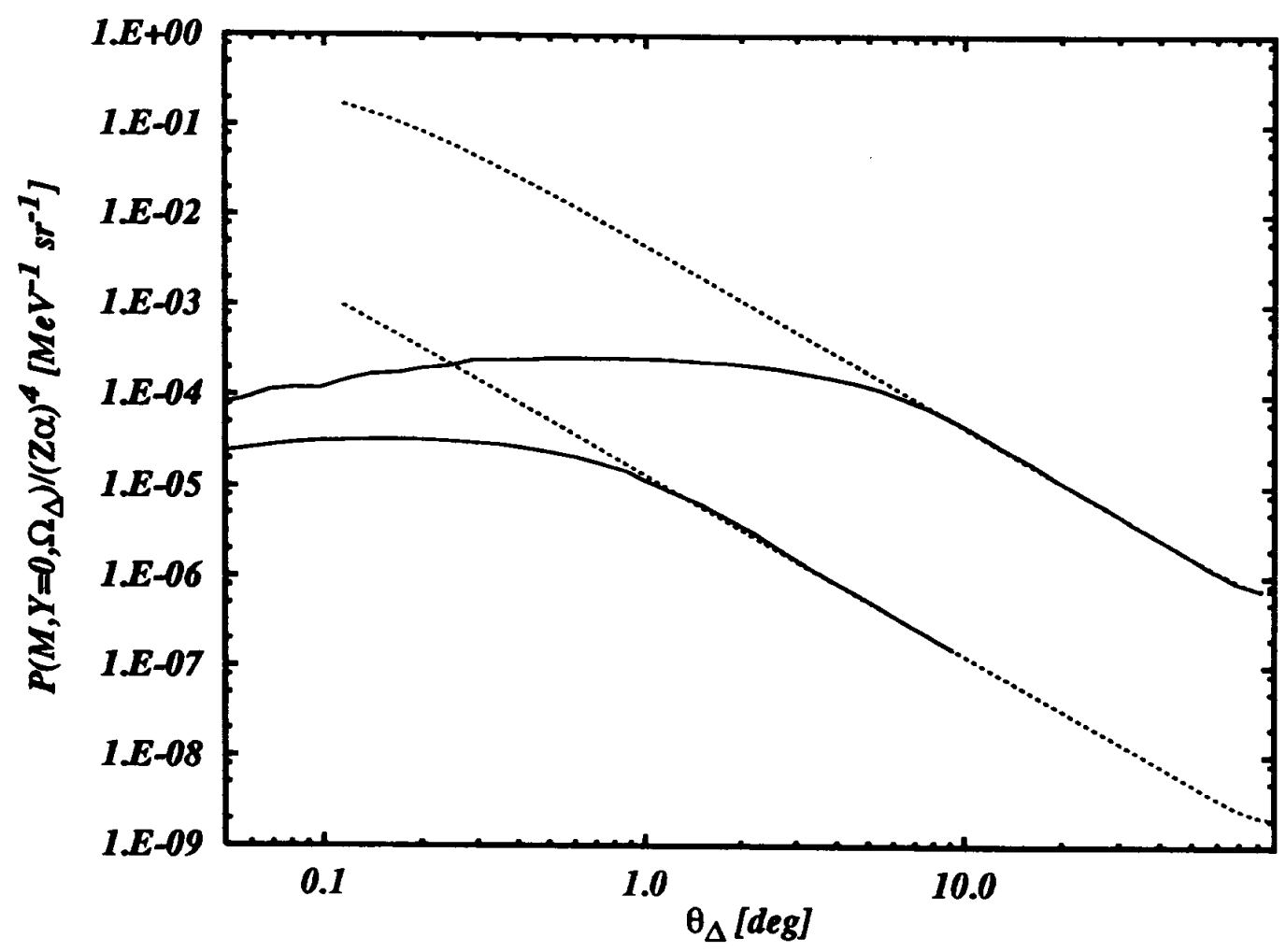

FIG. 15

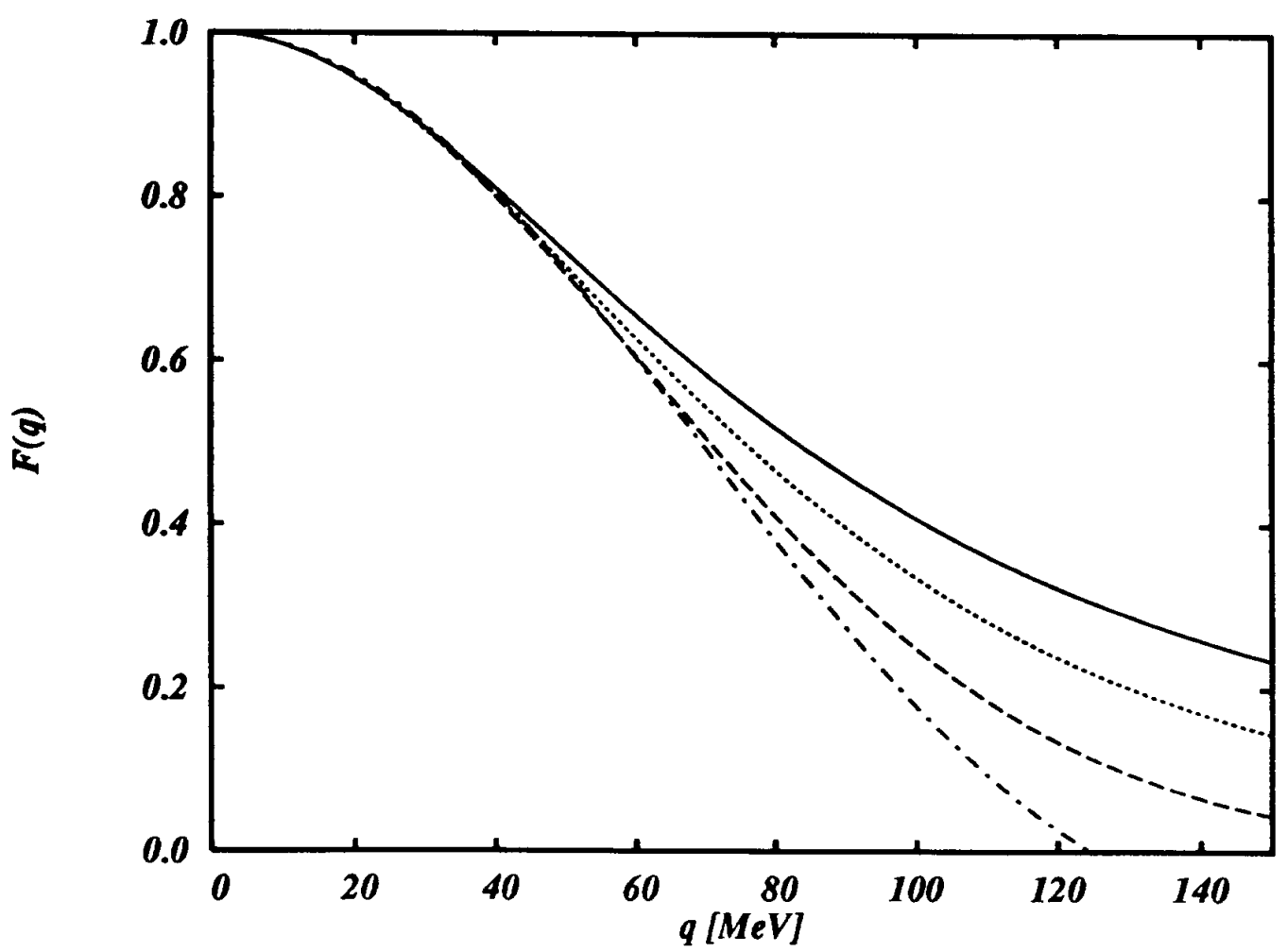

FIG. 16 
